\title{
Osteoponin Promoter Controlled by DNA Methylation: Aberrant Methylation in Cloned Porcine Genome
}

\author{
Chih-Jie Shen, ${ }^{1}$ Yung-An Tsou, ${ }^{1,2,3}$ Hsiao-Ling Chen, ${ }^{4}$ \\ Hung-Jin Huang, ${ }^{5}$ Shinn-Chih Wu, ${ }^{6}$ Winston T. K. Cheng, ${ }^{7}$ \\ Calvin Yu-Chian Chen, ${ }^{2,8,9,10}$ and Chuan-Mu Chen ${ }^{1,11}$ \\ ${ }^{1}$ Department of Life Sciences, National Chung Hsing University, Taichung 402, Taiwan \\ ${ }^{2}$ School of Medicine, College of Medicine, China Medical University, Taichung 40402, Taiwan \\ ${ }^{3}$ Department of Otolaryngology Head and Neck Surgery, China Medical University, Taichung 40402, Taiwan \\ ${ }^{4}$ Department of Bioresources, Da-Yeh University, Changhwa 515, Taiwan \\ ${ }^{5}$ Department of Chinese Pharmaceutical Sciences and Chinese Medicine Resources, College of Pharmacy, China Medical University, \\ Taichung 40402, Taiwan \\ ${ }^{6}$ Department of Animal Science and Technology, National Taiwan University, Taipei 106, Taiwan \\ ${ }^{7}$ Department of Animal Science and Biotechnology, Tung Hai University, Taichung 407, Taiwan \\ ${ }^{8}$ Department of Biomedical Informatics, Asia University, Taichung 41354, Taiwan \\ ${ }^{9}$ Department of Medical Research, Human Genetic Center, China Medical University Hospital, Taichung 40447, Taiwan \\ ${ }^{10}$ Research Center for Chinese Medicine \& Acupuncture, China Medical University, Taichung 40402, Taiwan \\ ${ }^{11}$ Rong Hsing Research Center for Translational Medicine and the iEGG Center, National Chung Hsing University, Taichung 402, Taiwan
}

Correspondence should be addressed to Calvin Yu-Chian Chen; ycc929@mit.edu and Chuan-Mu Chen; cmchen7010978@gmail.com

Received 24 February 2014; Accepted 5 March 2014; Published 2 July 2014

Academic Editor: Chung Y. Hsu

Copyright (C) 2014 Chih-Jie Shen et al. This is an open access article distributed under the Creative Commons Attribution License, which permits unrestricted use, distribution, and reproduction in any medium, provided the original work is properly cited.

\begin{abstract}
Cloned animals usually exhibited many defects in physical characteristics or aberrant epigenetic reprogramming, especially in some important organ development. Osteoponin (OPN) is an extracellular-matrix protein involved in heart and bone development and diseases. In this study, we investigated the correlation between OPN mRNA and its promoter methylation changes by the 5aza-dc treatment in fibroblast cell and promoter assay. Aberrant methylation of porcine $O P N$ was frequently found in different tissues of somatic nuclear transferred cloning pigs, and bisulfite sequence data suggested that the OPN promoter region -2615 to -2239 nucleotides (nt) may be a crucial regulation DNA element. In pig ear fibroblast cell culture study, the demethylation of OPN promoter was found in dose-dependent response of 5-aza-dc treatment and followed the OPN mRNA reexpression. In cloned pig study, discrepant expression pattern was identified in several cloned pig tissues, especially in brain, heart, and ear. Promoter assay data revealed that four methylated $\mathrm{CpG}$ sites presenting in the -2615 to $-2239 \mathrm{nt}$ region cause significant downregulation of $O P N$ promoter activity. These data suggested that methylation in the OPN promoter plays a crucial role in the regulation of $O P N$ expression that we found in cloned pigs genome.
\end{abstract}

\section{Introduction}

Nowadays, many of pathogenesis of diseases have been determined [1-3]. Methylation in the $5^{\prime}$ cytosine in the CpG dinucleotides is crucial a mechanism that regulates gene expression without changing DNA sequence and can be inherited to the offspring [4]. The promoter region contains various transcription factor binding motifs with numerous $\mathrm{CpG}$ dinucleotides. Some transcription factors are blocked by methylated $\mathrm{CpG}$ island resulting in inhibition of gene expression [5]. Somatic cell nuclear transfer (SCNT) technique is used to generate an identical genetic background offspring $[6,7]$. However, SCNT cloning animals usually showed low survival rate and impropriate methylation reprogramming 
process [8]. This dilemma of SCNT animal may be caused by methylation controlled genes, such as imprinting genes [9].

$O P N$ is an extracellular matrix protein and hydrophilic glycoprotein identified firstly in the bone as a sialoprotein. It contains a thrombin and transglutaminase cutting site, and the molecular weight is about $25 \mathrm{kDa}$ to $75 \mathrm{kDa}$; in pig, the molecular is about $67 \mathrm{kDa}$; it contains numerous isoforms [10]. OPN has a hydrophobic $N$ terminal; thus, it can be secreted out of cell membrane; the amino sequence of $O P N$ is full of Asp, Thr, and Ser that can elevate the binding activity with calcium, glycosylation, and phosphorylation, respectively [11]. Thus, OPN plays numerous roles in many aspects, such as bone remodeling, cell migration, iNOS regulation, repairment, and leucocyte recruitment [12]. And acquired $O P N$ expression has been found in a variety of cancer cell types, especially in the liver, lung, breast, prostate, colon, brain, and spleen $[13,14]$. OPN is cleaved by MMPs protein to generate functional OPN that can bind to $\alpha \mathrm{v} \beta 3$ [15]. This integrin binding with $O P N$ has influence on $\mathrm{NF} \kappa \mathrm{B}$ signaling transduction $[16,17]$. Therefore, overexpressed $O P N$ is associated with tumorigenesis, tumor invasion, and metastasis $[18,19]$. Previous study suggested that overexpressed OPN induces the serious cardiac fibrosis $[20,21]$. Thus, our cloned pigs were also surrounded by various defects in heart fibrosis and retardation of growth of bones. Therefore, this study focuses on the methylation change of OPN promoter that may be disrupted by inappropriate reprogramming process. Consequently, aberrant methylation of promoter could lead to aberrant expression of OPN. In the previous studies, OPN expression was induced with TSA (trichostatin A) in mouse undifferentiated mesenchymal cell line by AP1 site [22]. The TSA is a histone deacetylase inhibitor. It can lose the chromatin structure in order to let gene restore its expression. 5 -aza-dc is also an analog with the same structure of cytosine without methyl group adding in the $5^{\prime} \mathrm{C}$ end [23].

Thus, 5-aza-dc addition leads to low methylation percentage in the $\mathrm{CpG}$ sites rich region. The hypomethylation status in the promoter may contribute its gene transcription activity. Porcine fetal fibroblasts in 5th passage cultures were treated with $0.5,1.0,2.0$, and $3.0 \mu \mathrm{M} 5$-aza-dc for $96 \mathrm{~h}$; 5-azadc inhibited the growth of cell at all concentrations. 5-aza$\mathrm{dc}$ induced a reduction of transcripts level in DNMT1 and increasing expression in imprinted gene, IGF2 [24]. Furthermore human $O P N$ promoter sequence is similar to porcine in the front $400 \mathrm{nt}$ of the porcine promoter. Therefore, we investigated OPN RNA and promoter methylation changes in the porcine ear fibroblast cell. Data showed that the elevated OPN expression and in 5-aza-dc treated fibroblast cell is due to the decreased methylation of $O P N$ promoter. Cloned pigs samples had found extremely methylation changes, especially in the brain (99.75\% upregulation), heart (11.50\% downregulation), and ear (18.03\% down-regulation). Deletion analysis of the promoter region revealed 5-aza-dc induced luciferase response that was regulated by -2615 to -2239 of the $O P N$ promoter. These data suggested that methylation in the OPN promoter plays a crucial role in the regulation of $O P N$ expression. Methylation of $O P N$ promoter may be an epigenetic marker of diagnosis of cancer.

\section{Materials and Methods}

2.1. CpG Island Prediction. The sequence of a putative $\mathrm{CpG}$ island in $O P N$ promoter was analysed by using MethPrimer software (http://www.urogene.org/methprimer/indexl.html).

2.2. Cell Culture. The porcine fibroblast cell line was grown in Dulbecco's modified Eagle's medium (DMEM; GibcoBRL, Gaithersburg, MD, USA) supplemented with 10\% fetal bovine serum (FBS, Gibco BRL) and containing $100 \mathrm{U} / \mathrm{mL}$ penicillin and streptomycin. The cells were incubated at $37^{\circ} \mathrm{C}$ in humidified incubator with $5 \% \mathrm{CO}_{2}$.

2.3. 5-aza-dc Demethylation Drug Treatment. For 5-aza-dc treatment, porcine fibroblast cell in 5th passage cultures was treated with 5 -aza-dc (sigma) at various concentrations, that is, 0 (control), $0.5,1.5$, and $2.0 \mu \mathrm{M}$, for $72 \mathrm{~h}$. Medium was changed every $24 \mathrm{~h}$ and then cells were collected for RNA and DNA extraction and stored at $-80^{\circ} \mathrm{C}[24]$.

2.4. Quantitative Real Time-PCR. $2 \mu \mathrm{g}$ RNA of ear fibroblast cell was used to be transformed to cDNA. $0.5 \mu \mathrm{L}$ of cDNA was performed for quantitative real time-PCR with RotorGene 6000 (Corbett). $\beta$-actin was the internal control for normalize target gene, OPN. The calculated gene expression fold from CT value was according to the previous study. $P$ value less than 0.5 exhibited the obviously significant difference.

2.5. Methylation Analysis by Combined Bisulfite Restriction Analysis (COBRA). For amplification of porcine OPN promoter methylation analysis site, PCR was performed using $2 \mu \mathrm{L}$ of bisulfite-converted genomic DNA as template. The primer sets of COBRA were OPN-C sense $5^{\prime}$ TTTTTTGAGGGAGATTAGTTTTTG- $3^{\prime}$ and antisense $5^{\prime}$ ATTCTACTAAAATCCAACCACCC- $3^{\prime}$. The COBRA-PCR products were purified by phenol/chloroform, followed by ethanol precipitation. The DNA was resuspended in $8.5 \mu \mathrm{L}$ of distilled deionized water. Purified PCR products were then digested with $10 \mathrm{U}$ BstUI restriction enzyme (New England Biolabs, MA, USA) at $65^{\circ} \mathrm{C}$. Products were electrophoresed on $6 \%$ native acrylamide gel, stained with $200 \mathrm{~g} / \mathrm{mL}$ ethidium bromide, and visualized using a Kodak 1D software.

2.6. Methylation Specific-PCR. Genomic DNA $(0.5 \mu \mathrm{g})$ was treated with sodium bisulfite according to the manufacture's recommendations (EZ DNA Methylation Kit; Zymo research, CA, USA) and amplified with specific primers for methylated or unmethylated DNA. The primer sets of MS-PCR were OPN-M sense $5^{\prime}$-AAGCGGGGAAGGAGTTATTACGT$3^{\prime}$, antisense $5^{\prime}$-TCCGACAAAACGAAACGATCATACA-3', OPN-U sense $5^{\prime}$-GAAGTGGGGAAGGAGTTTATTATGT$3^{\prime}$, and antisense $5^{\prime}$-CAATAACTCCAACAAAACAAAACAATC$3^{\prime}$. All PCR reactions were performed on PTC 200 thermocyclers (MJ Research, MA, USA) and in $25 \mu \mathrm{L}$ volume using the PlatiumTaq DNA polymerase system (Invitrogen, CA, USA). PCR products were separated on $1.5 \%$ agarose gels. The $\mathrm{M}$-set primers contained at least three 
CpG sites to distinguish the methylation status of investigated region. And U-set primers overlapping the $\mathrm{M}$-set primers were used to amplify the unmethylated region.

2.7. Plasmid Constructs. A full length pig OPN promoter (-2615-luc) was amplified from wild-type pig heart tissue cDNA. This fragment was cloned into a luciferase fusion plasmid, pGL3-Enhancer vector (Promega), to generate pOPNfull-luc. HindIII and NcoI cutting sites were used for cloning. Three truncated forms of pOPN promoter were prepared by PCR using the pOPN-full-luc as a template and using synthesized oligonucleotides as follows: pOPN-full-luc: sense, $5^{\prime}$ AAGCTTGAATTCACTCGTCTTTCCTTTGAGA-3', and antisense, $5^{\prime}$-CCATGGGCTGACAGCCTGGACCTCCCC3'; -2239-luc: sense, $5^{\prime}$-AAGCTTCCTATAACTGTCTACGTTCATATTAGAC- $3^{\prime}$, and antisense, $5^{\prime}$-CCATGGGCTGACAGCCTGGACCTCCCC-3' ; -1505-luc: sense, $5^{\prime}$-AAGCTTAATTTTCATTTAAGTAACCAACTTTATATATC$3^{\prime}$, and antisense, $5^{\prime}$-CCATGGGCTGACAGCCTGGACCTCCCC-3'; -495-luc: sense, $5^{\prime}$-AAGCTTGCCTGAACAATATAGCCTTGTCGC-3', and antisense, $5^{\prime}$-CCATGGGCTGACAGCCTGGACCTCCCC- $3^{\prime}$. The sequence of constructs was confirmed by DNA sequencing. There were twopoint mutation different from NCBI: one is $287 \mathrm{~A}$ to $\mathrm{T}$ and the other is $957 \mathrm{~T}$ to $\mathrm{A}$.

2.8. Transient Transfection and Luciferase Assay. Pig ear fibroblast cells were transfected using the Lipofectamine 2000 (Invitrogen). Fibroblast cells were incubated at a density of $8 \times 10^{5}$ cells into $35 \mathrm{~mm}$ diameter dishes. After $24 \mathrm{~h}$ when cell was adherent to the dishes, $3 \mu \mathrm{g}$ of reporter plasmid DNA was transfected for $6 \mathrm{~h}$ in Lipofectamine mixture (Invitrogen). $24 \mathrm{~h}$ after the transfection, cell lysates were collected for a luciferase assay. The luciferase activity of the cell lysates was detected by Dual-light system (Applied biosystems). The activity data was measured with PARADIGM Detection Platforms (Beckman Coulter). Luciferase activity was normalized with $1 \mu \mathrm{g} \beta$-gal plasmid. All luciferase assays were carried out in triplicate.

2.9. In Vitro Methylation of the OPN Promoter Region. The OPN reporter construct -495 -luc was methylated by incubation with SssI methyltransferase (New England BioLabs). The -2615-luc construct was methylated by HhaI and HpaII methyltransferase (New England BioLabs) for $16 \mathrm{~h}$ at $37^{\circ} \mathrm{C}$. The methylation status was also verified by digested with HhaI and HpaII enzyme.

2.10. Electrophoresis Mobile Shift Assays. Nuclear extracts were prepared from HEK293T cells. Two probes were designed for methylation binding activity test. Two probes containing either 6-8 CpG sites or 13 th $\mathrm{CpG}$ site in the -2615 to -2239 of the $O P N$ promoter were generated by annealing two complementary oligonucleotides

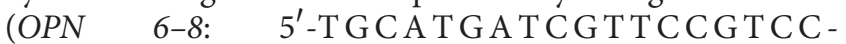
TGCCGGAGTCACTGACGGAACCAGACCGAGGT-3’; $5^{\prime}$-ACCTCGGTCTGGTTCCGTCAGTGACTCCGGCAGGACGGAACGATCATGCA-3', the predicted core

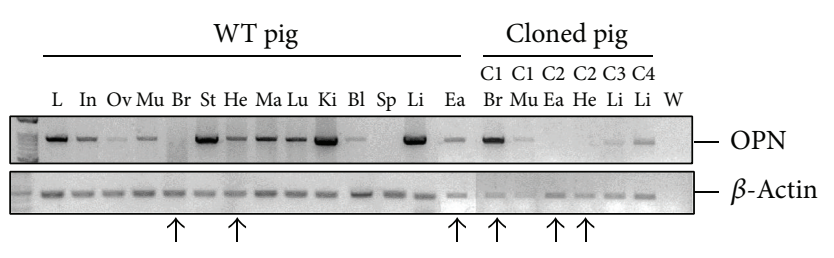

(a)

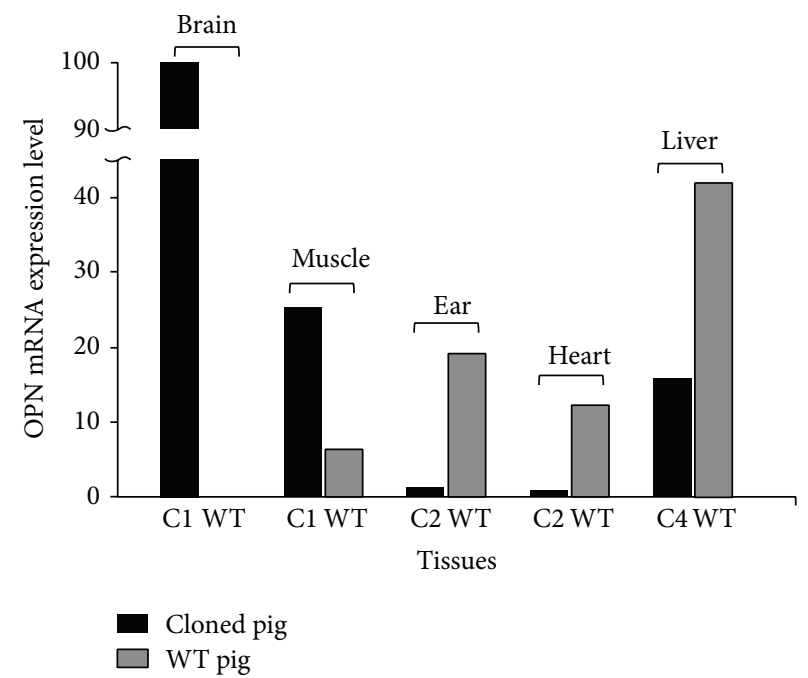

(b)

FIGURE 1: (a) (b) The semiquantitative RNA expression of OPN. $\mathrm{C} 1-\mathrm{C} 4$ indicated four different cloned pigs. The black arrows represent the contrary expression in the cloned pig tissues relative to WT tissues. Br: brain, Ea: ear, He: heart, Ki: kidney, Li: liver, Lu: lung, Mu: muscle, Sk: skin, In: intestine, Sp: spleen, Pl: placenta, Um: umbilical cord, B: blood; S: blood treated with SssI, and W: $\mathrm{ddH}_{2} \mathrm{O}$.

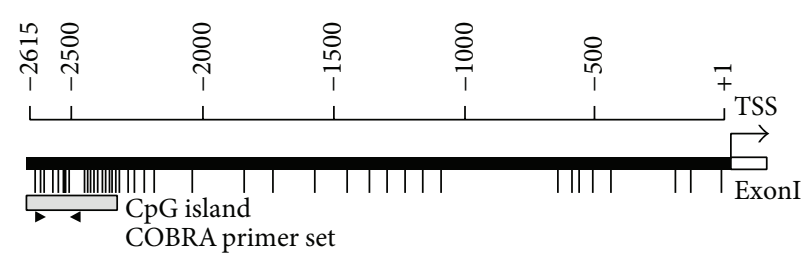

(a)

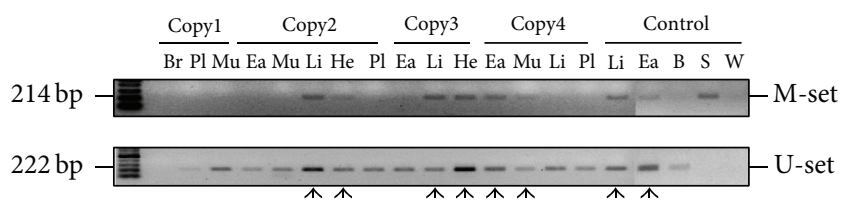

(b)

FIGURE 2: MS-PCR analysis of OPN promoter. (a) The distribution of $\mathrm{CpG}$ sites in the pig OPN promoter. The two arrows indicated the MS-PCR primer sets. (b) The MS-PCR results of OPN promoter in cloned pigs. The arrows represent both existence of methylation and unmethylation DNA element. Br: brain, Ea: ear, He: heart, Li: liver, Lu: lung, Mu: muscle, Pl: placenta, B: blood; S: blood treated with SssI, and W: $\mathrm{ddH}_{2} \mathrm{O}$. 

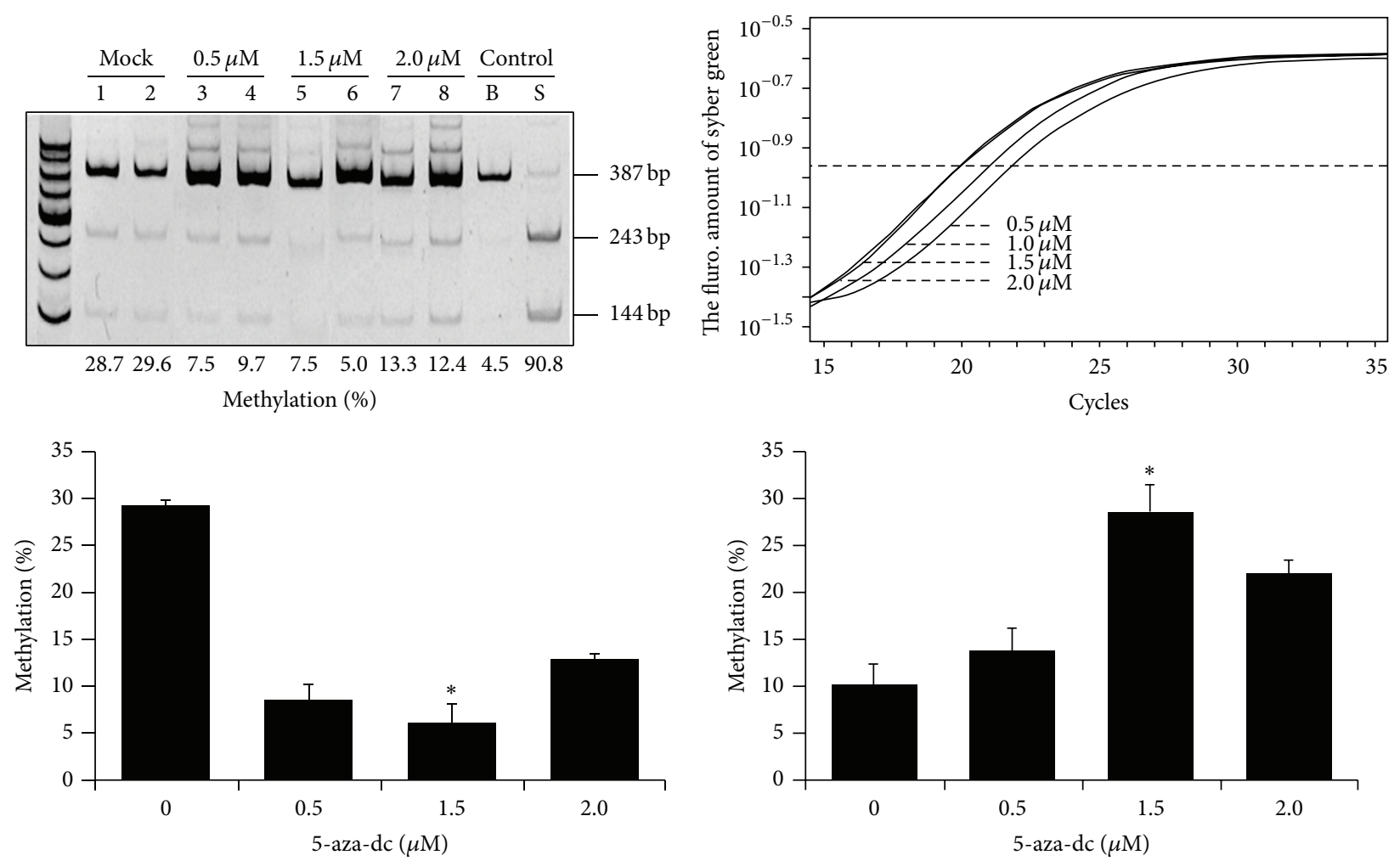

(a)

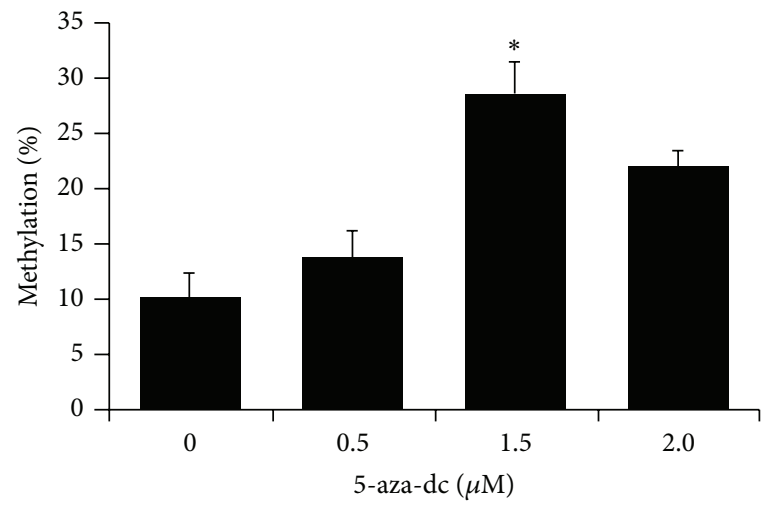

(b)

FIGURE 3: OPN RNA expression and DNA methylation of 5-aza-dc treated pig fibroblast cell. (a) The schematic showed the methylation percentage and mRNA expression of OPN promoter in different concentrations 5-aza-dc. (b) The COBRA analysis of OPN promoter. Below the square is the methylation percentage of $O P N$ promoter methylation.

sequence of the AP1 binding site is underlined; OPN13th: $\quad 5$ '-CCTCCGTGTTCCCTGTTAATGTGT AGCGCGTCGTTGTTGGGAAATAGTTC-3 ; $5^{\prime}$ GAACTATTTCCCAACAACGACGCGCTACACATTAACAGGGAACACGGAGG- $3^{\prime}$; the predicted core sequence of the ADR1 binding site is underlined). The transcription factor prediction software is TFSEARCH 3.0 version. The probes were labeled with $\gamma-{ }^{32} \mathrm{P}$-ATP by using T4 kinase (Promega). Annealing probes also were methylated with SssI methyltransferase (NEB). Nuclear extracts containing $5.6 \mu \mathrm{g}$ of the protein were preincubated in $20 \mu \mathrm{L}$ of binding buffer $(50 \mathrm{mM}$ Tris- $\mathrm{HCl}(\mathrm{pH} 8.0)$, $750 \mathrm{mM} \mathrm{KCl}, 2.5 \mathrm{mM}$ EDTA, $0.5 \%$ Triton-X 100, 62.5\% glycerol $(\mathrm{v} / \mathrm{v})$, and $1 \mathrm{mM}$ DTT) with or without unlabeled competitor (10-fold molar excess). For supershift assay, antibody of AP1 was added to the preincubation buffer. After 10 min of preincubation on ice, the DNA probe labeled with $\left[\gamma-{ }^{32} \mathrm{P}\right]$-ATP was added, and the mixtures were incubated at room temperature for $30 \mathrm{~min}$. The reaction mixtures were resolved on $6 \%$ polyacrylamide gels. The gels were dried and subjected to PhosphorImager analysis using a Typhoon system and ImageQuant TL software (Amersham Biosciences, Sunnyvale, CA, USA).

2.11. Molecular Modelling. The molecular docking was then further analyzed for proving the further mechanism of our findings; we further surveyed the interaction of AP1 (c-Jun) and TFIIB by computational biology. The AP1 (c-Jun) is possible higher spot for hypermethylation in OPN promoter area and provides the binding domain for RNA-polymerase II initial binding transcription factor (TFIIB) in this study. Therefore, we first utilized the Z-DOCK program to simulate the structures of c-Jun and TFIIB. After that, we further used molecular dynamics (MD) to validate the stability of c-Jun and TFIIB complex under the GROMACS 4.5.5 program [25] with charmm27 force field. The model is set in the TIP3P water modeling in $1.2 \mathrm{~nm}$ distance of box for water box setting. $\mathrm{Na}$ and $\mathrm{Cl}$ ions in the concentration of $0.145 \mathrm{M} \mathrm{NaCl}$ model are used for system neutralization. All bonds are fixed by linear constraint solver (LINCS) algorithm to constrain all bonds lengths in the simulation system. Newton's Law is utilized for calculating the motion of molecular dynamics as follows:

$$
\frac{d^{2} r}{d t}=M^{-1} F
$$

The Particle mesh Ewald (PME) is also used for calculate the coulomb type of electrostatics. The Van der Waals (VDW) interactions are set as $1.4 \mathrm{~nm}$ cut-off distance for nonbound interaction. The first step is set on the 5,000 cycle steps performed in the manner of Steepest Descent algorithm for energy minimization. And then, equilibration was performed 


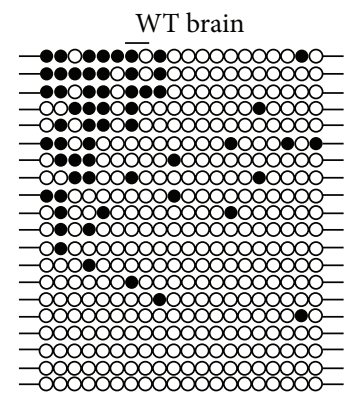

Total $57 / 400=14.3 \%$

CpG1-10 49/200 = 24.5\%

Copy1 brain

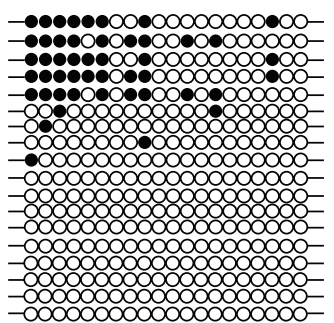

Total $57 / 400=13.3 \%$

CpG1-10 49/200 = 22.2\%

Copy5 brain

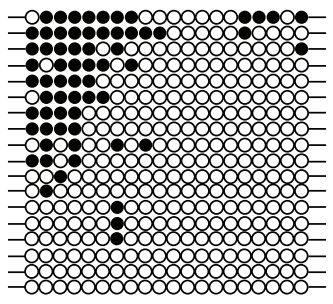

Total $57 / 400=18.1 \%$

CpG1-10 49/200 = 32.8\%
WT heart

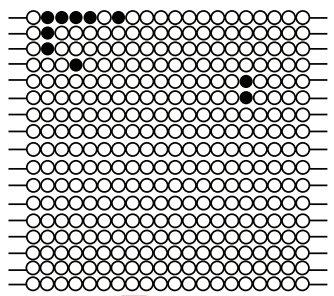

Total $57 / 400=2.9 \%$

CpG1-10 49/200 = 4.7\%

Copy2 heart

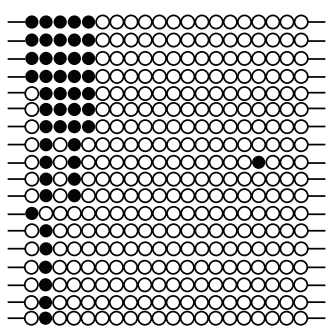

Total $57 / 400=13.3 \%$

CpG1-10 49/200 = 26.1\%

Copy3 heart

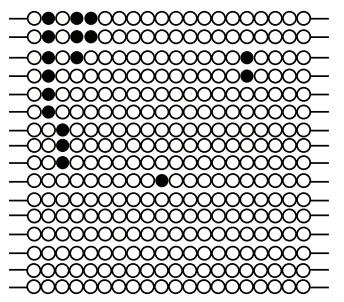

Total 57/400 $=5.3 \%$

CpG1-10 49/200 = 9.4\%

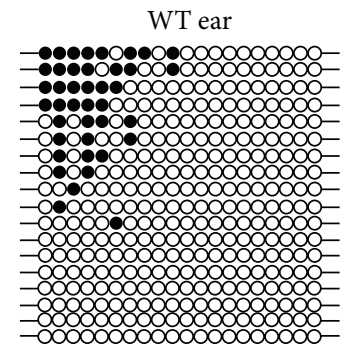

Total $57 / 400=11.7 \%$

CpG1-10 49/200 = 23.3\%

Copy2 ear

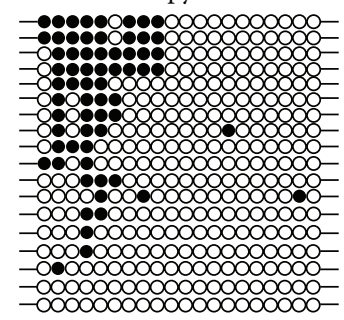

Total $57 / 400=18.1 \%$

CpG1-10 49/200 = 35\%
WT liver

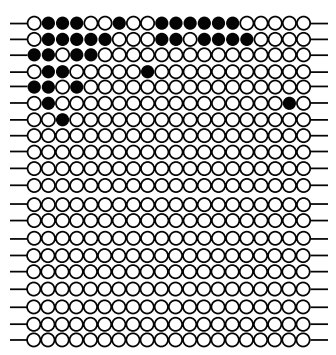

Total $57 / 400=8.5 \%$

CpG1-10 49/200 = 11.5\%

Copy4 liver

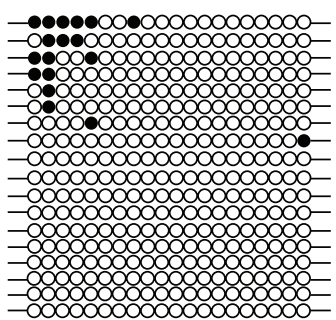

Total 57/400 = 5.0\%

CpG1-10 49/200 = 9.4\%

FIGURE 4: The bisulfite sequencing of $O P N-2610 \sim-2400 \mathrm{nt}$ upstream the promoter in cloned pigs' tissues. The closed circles represent the methylation CpG sites. The hollow circles represent the unmethylated CpG sites. The bottom number indicated the methylation percentage of each sample. The range of square showed that the region may be the methylation controlled region of OPN promoter.

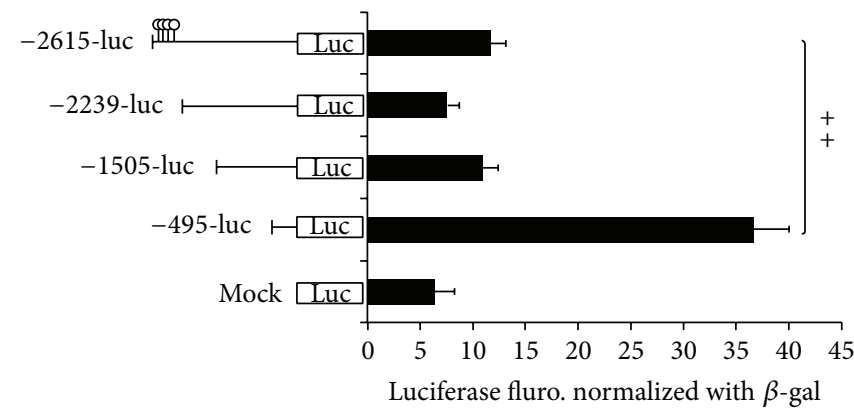

(a)

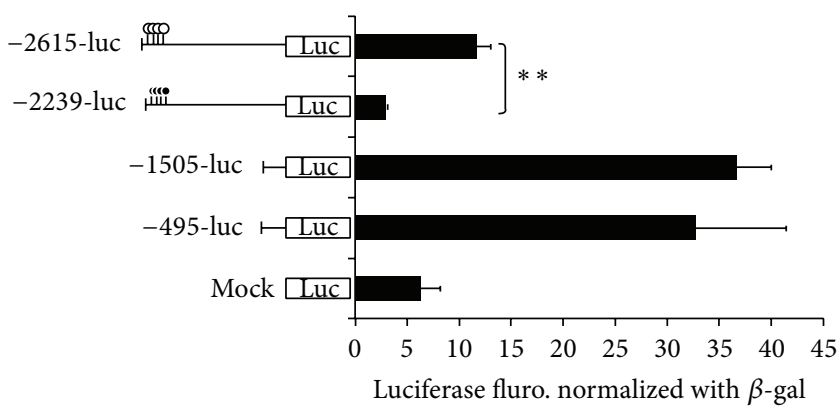

(b)

FIGURE 5: Methylation and deletion analysis of OPN promoter in 293T cells. The match-like bar with black circle represents the methylation CpG site; white circle of match bar indicated the unmethylation CpG sites. PGL3 vector as standard; in 293T cell line; PGL3-enhance vector as negative control; cell lysate as the background; pCMV-b-gal as internal control. The relative value is adjusted by cell lysate; $-495 \mathrm{M}-l$ uc indicated the methylation in PGL3 backbone with HhaI and HpaII methyltransferase. $\left({ }^{* *} P<0.01\right)$; -2615 -luc and $-2615 \mathrm{M}-\mathrm{luc}: n=3$; -495 -luc and -495 M-luc: $n=4$. The experiments were repeated three times and the results were analyzed and presented as the mean \pm SE. 


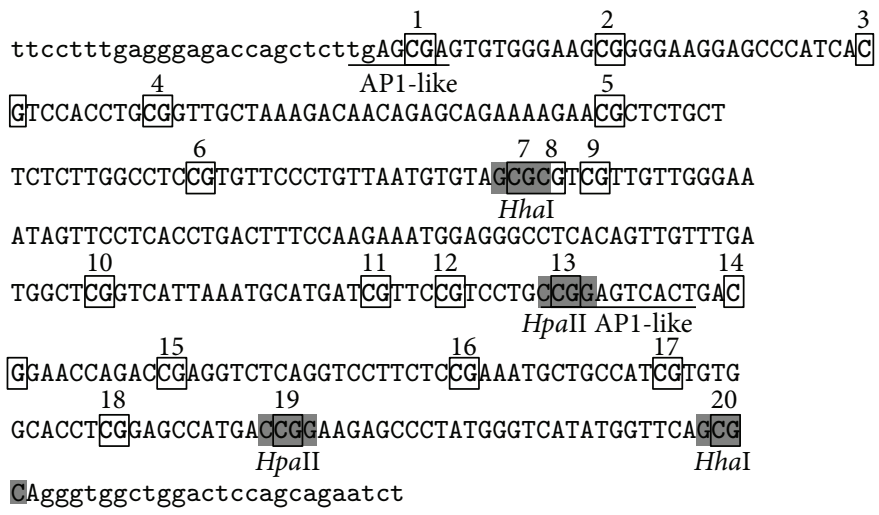

Figure 6: The sequence and CpG sties distribution of pig OPN promoter. $20 \mathrm{CpG}$ sites exist in the front region of OPN promoter. The underline indicates the predicted AP1-like binding site. The gray marker indicates two methyltransferase sites of HhaI (GCGC) and HpaII (CCGG).

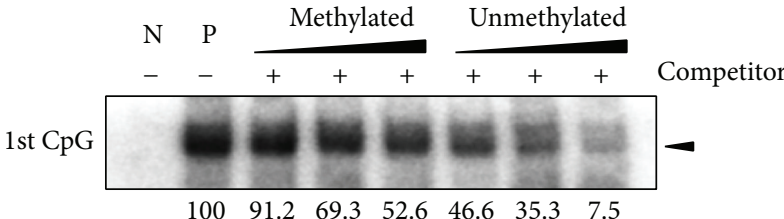

(\%)

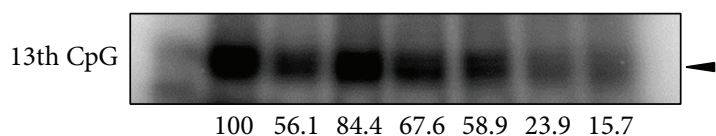

(\%)

(a)

(b)

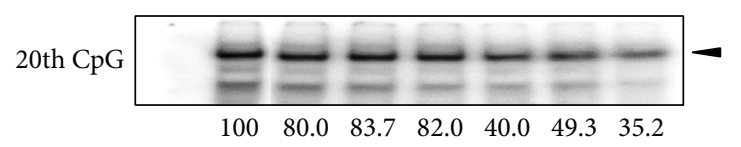

(\%)

(c)

FIGURE 7: Electrophoresis mobile shift assay in porcine OPN promoter CpG sites. (a) Using CpG1 contained DNA element binding with SH-SY5Y nuclear extract. Methylated and unmethylated competitors were used as $2 \mathrm{X}, 5 \mathrm{X}$, and 10X concentration than isotope labeled probe. (b) CpG 13th probe of OPN promoter binding with HEK-293 nuclear extract. (c) CpG 20th of OPN promoter element binding with HEK-293 nuclear extract. There was no difference between methylated or unmethylated competitors in competition experiment.

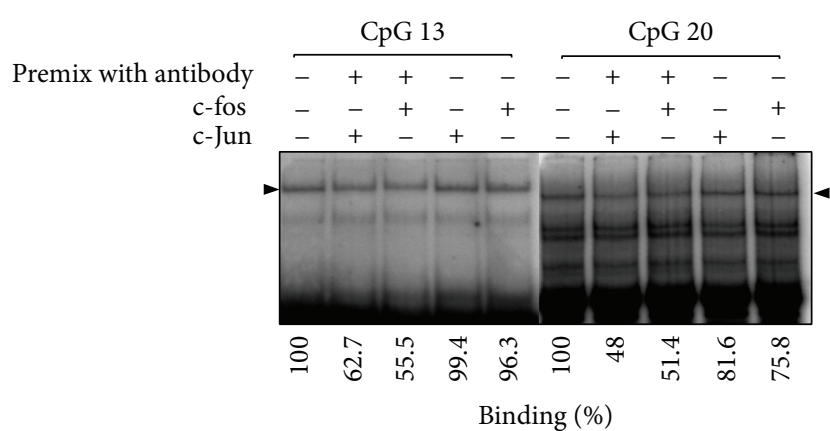

Figure 8: The CpG 13 and CpG 20 showed competition binding activity characteristics with $\mathrm{c}$-Jun and c-Fos. The competition reduced the binding activity between transcription factors and labeled OPN EMSA probe. The amount of EMSA c-Jun and c-Fos antibody is $3 \mu \mathrm{g}$. The EMSA probe is $30 \mu \mathrm{g}$. Adding the $\mathrm{Ab}$ with a premix way can reduce the binding effects with the transcription factors. It is suggested that the premix with antibody blocks the access to its binding site (antibody-transcription factor-DNA). with a time period of $1 \mathrm{~ns}$ for position restraints set under the constant temperature dynamics (NVT type) conditions. The third step is calculating the production run for $5000 \mathrm{ps}$ under constant pressure and temperature dynamics (NPT type). All the MD systems are set by $310 \mathrm{~K}$ temperature during all simulation times. MD frames data were saved every $20 \mathrm{ps}$ for all production runs.

2.12. Molecular Dynamics Analysis. First we survey the stability of all atoms performed by using the GROMACS 4.5.5 software though the commands of g_rms and g_gyrate to calculate root mean square deviation (RMSD) and radius of gyration $(\mathrm{Rg})$, respectively. Secondly, we calculate the total energy for all the systems by the command of g_energy. Thirdly, we further calculate root mean squared fluctuation (RMSF) for each protein residue by commands of g_rmsf. Fourthly, the distance between c-Jun and TFIIB and movement analysis are calculated by the g_dist program. Fifthly, the migration of dock protein (c-Jun and TFIIB) is 


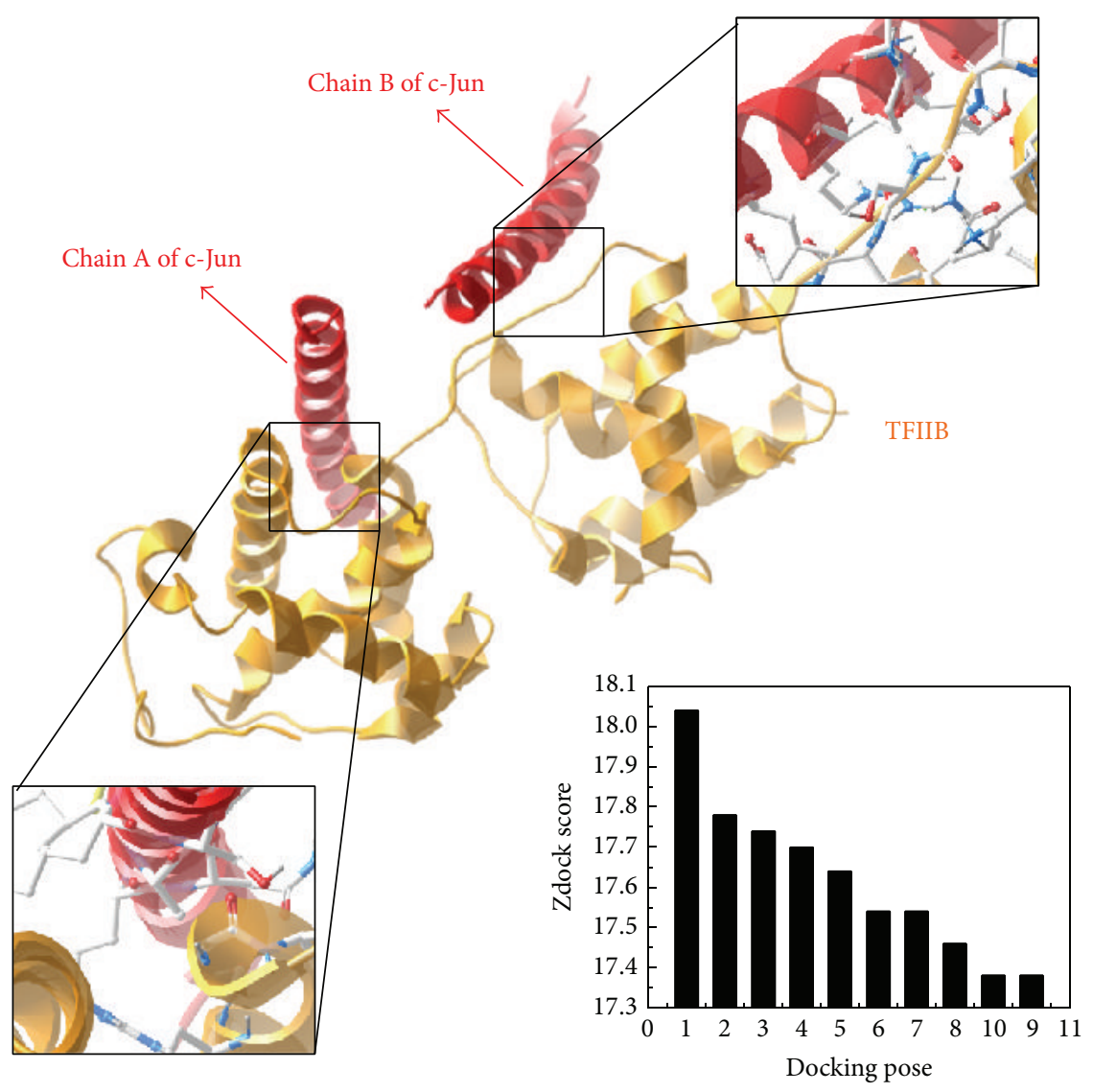

FIGURE 9: The best docking pose of c-Jun and TFIIB with 18.04 Zdock Score. The structures of c-Jun and TFIIB are colored in red and orange, respectively.

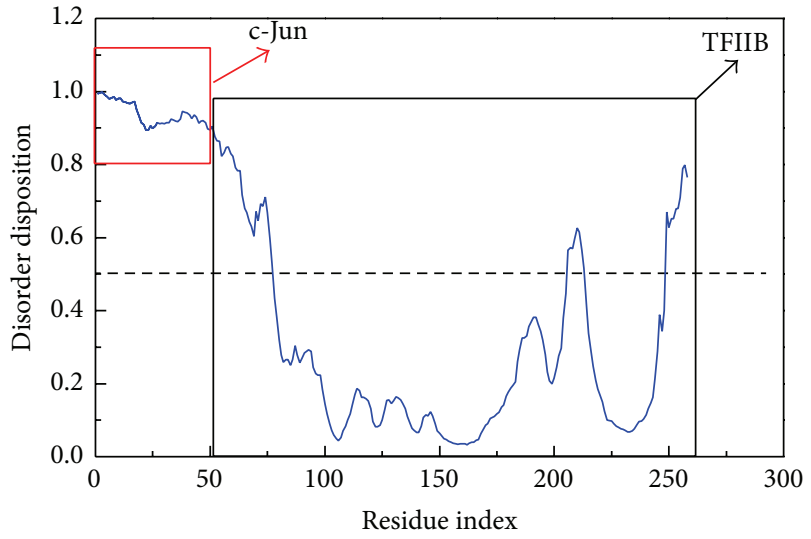

FIGURE 10: Disorder prediction of c-Jun and TFIIB complex; the value of disorder disposition below 0.5 indicates order folding region. The sequence of c-Jun is in the region from residue index 0 to 54 , and the sequence of TFIIB is in the region from residue index 55 to 258 . The folded structure of $\mathrm{c}$-Jun reveals disorder; the N-terminal and C-terminal of TFIIB structure display folded disorder.

presented by mean square displacement (MSD) under the command of g_msd module in GROMACS during all the simulation times. Sixthly, the g_cluster program is selected for further calculation of the representative structure from all MD frames, and the representative structure is taken for further snapshot analysis. We also employed DSSP analysis and matrices of the smallest distances between each residue to investigate the stability of the protein structure. The principle component analysis (PCA) is then applied to observe the protein motion changes during all the MD frames. Finally, in order to observe the compactness between c-Jun and TFIIB, Caver 3.0 software [26] was used to predicted space in the complex.

\section{Results}

3.1. Distribution of Porcine OPN CPG Island. There are dense $\mathrm{CpG}$ sites existing in the front of the OPN promoter region. One putative $\mathrm{CpG}$ island was found $(\mathrm{CpG}$ island size $>100$, GC Percent $>50.0$, Obs/Exp $>0.6$ ) by MethPrimer program.

3.2. Methylation and Expression Analysis of OPN in Cloned Pig Various Tissues. Firstly, the OPN mRNA expression was investigated in the WT pig tissues and cloned pig tissues. Data showed the various expression levels in different tissues. Particularly, Copyl brain overexpressed the OPN and Copy2 ear with no expression of $O P N$ relative to its wild-type tissue, respectively (Figure 1). The unique aberrant 


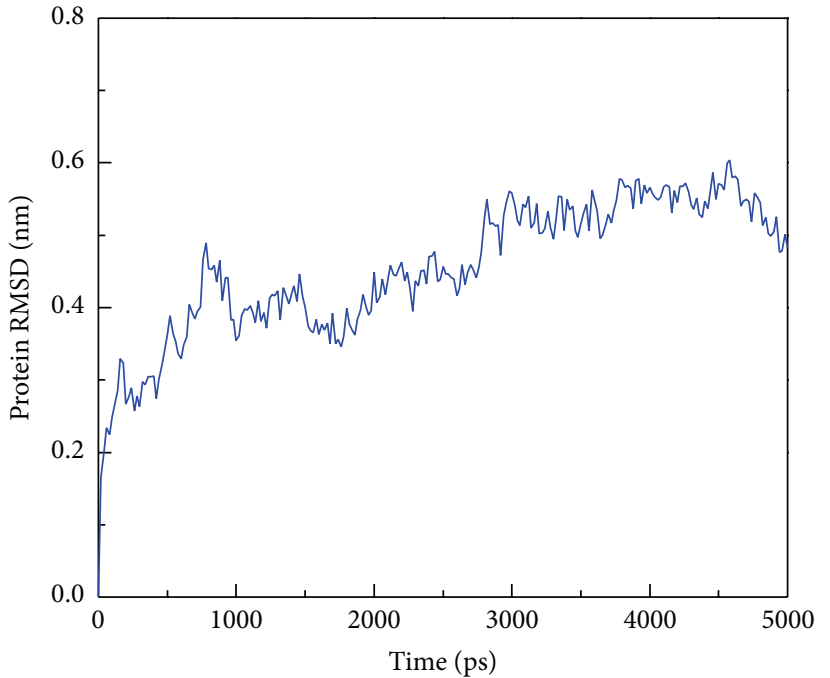

(a)

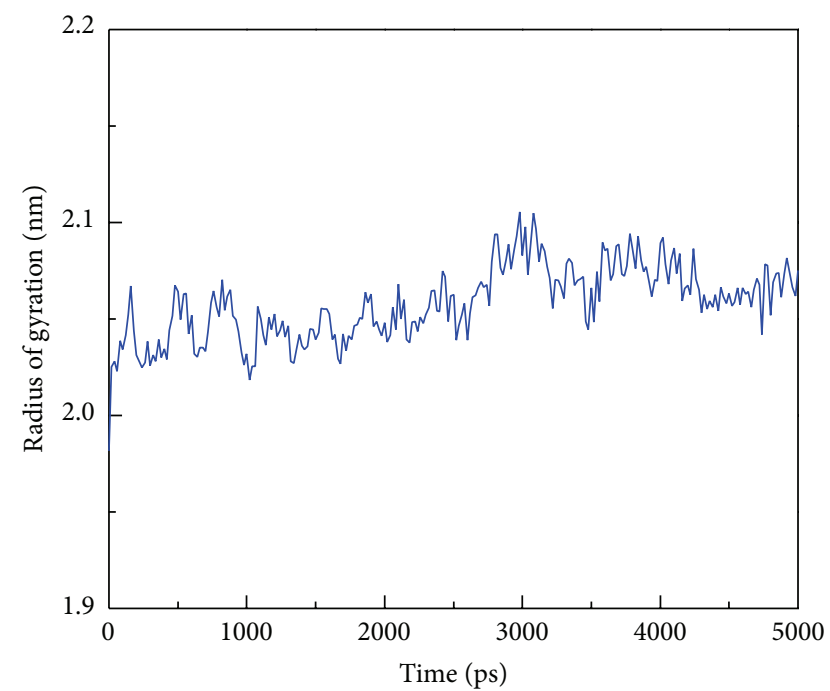

(b)

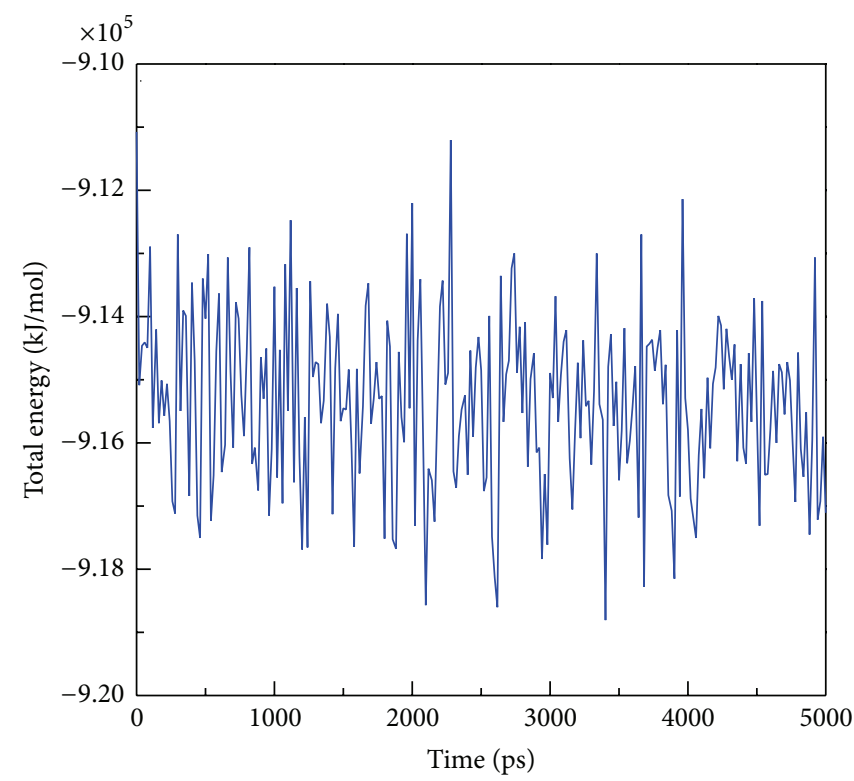

(c)

FIGURE 11: The trajectory analysis of c-Jun and TFIIB during 5000 ps simulation times. (a) RMSD values of all atoms of c-Jun and TFIIB complex; (b) radius of gyration of c-Jun and TFIIB complex for identifying the compactness of protein structure; (c) total energy of all simulated systems of c-Jun and TFIIB complex; the total energy is sum of potential energy and kinetic energy.

expression patterns exhibited the different control way of the $O P N$ expression. We proposed that $O P N$ expression may be a tissue-specific manner. MS-PCR primers were designed to estimate the methylation status of $O P N$ promoter. Hypomethylation generally appeared in the various tissues of cloned pigs. However, there were still some tissues that showed the methylated region in OPN promoter (Figure 2).

3.3. 5-aza-dc Increases OPN mRNA and Decreases Methylation of OPN Promoter in Pig Ear Fibroblast Cell. In order to realize whether the methylated $O P N$ promoter affects the activity of OPN promoter. The 5-aza-dc treated porcine ear fibroblast cells showed that when the concentration of 5-aza$\mathrm{dc}$ level increased, it will decrease the methylation of $O P N$ promoter and restore the OPN RNA expression at 0.5 to $2.0 \mu \mathrm{M}$ (Figure 3). The results suggested that the activity of OPN promoter can be affected by DNA methylation directly or indirectly. COBRA assay was also used to investigate the methylation status of OPN promoter in WT tissues. Brain, ear, liver, and lung tissues exhibited little part methylation of the $O P N$ promoter (data not show). This is thought that the methylation of $O P N$ promoter in the aforementioned tissues may be involved with $O P N$ transcript regulation mechanism. 


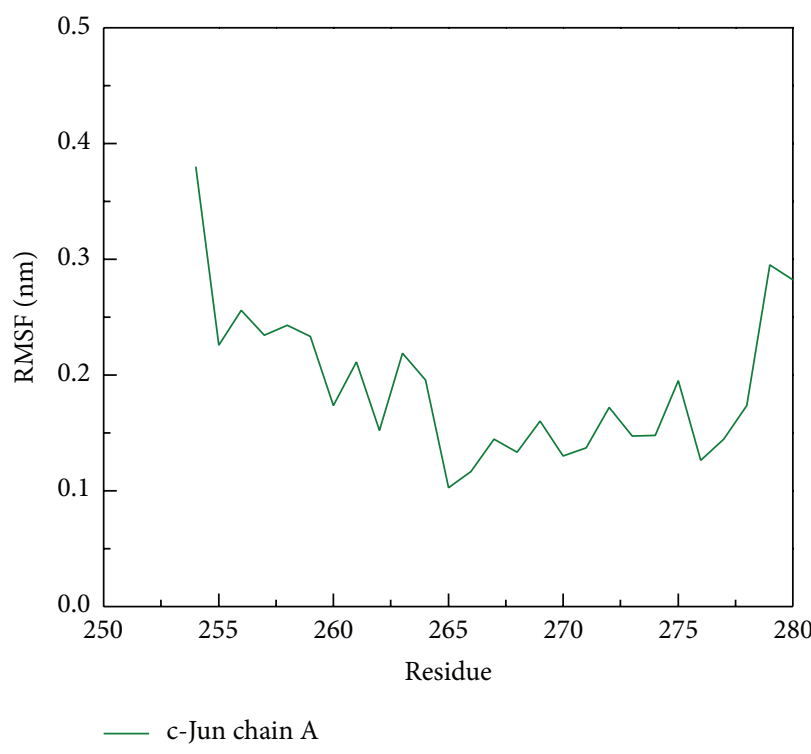

(a)

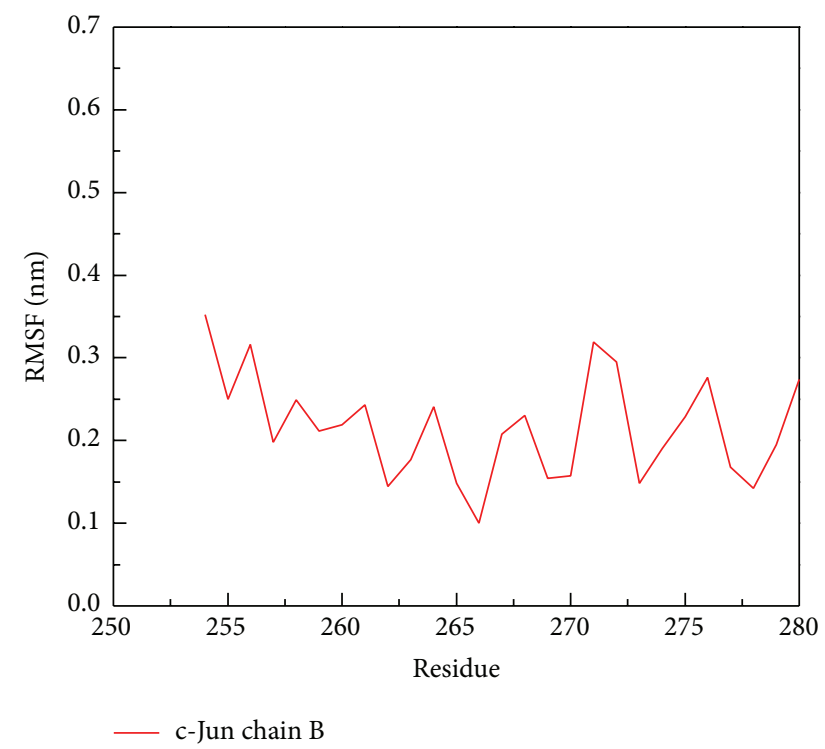

(b)

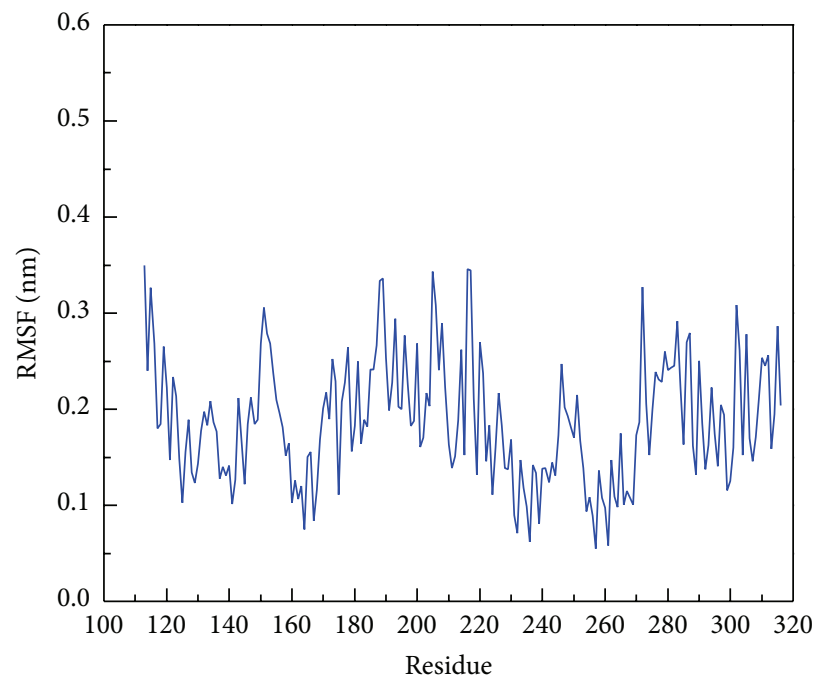

TFIIB

(c)

FIGURE 12: RMSF analysis of protein resides on (a) chain A of c-Jun, (b) chain B of c-Jun, and (c) TFIIB during simulation time of 5000 ps. The residue index of chain A and chain B of c-Jun is from 254 to 280, and the residue index of TFIIB is from 113 to 316 . The high values of RMSF indicated the high fluctuation of residue during all simulation times.

3.4. Bisulfite Sequencing Analysis of the Whole CpG Sites Methylation Profile in Cloned Pigs. To investigate which region of the OPN promoter is affected by methylation in the $\mathrm{CpG}$ site, bisulfite sequencing was performed to dissect the methylation status of $\mathrm{CpG}$ sites in the $O P N$ promoter. In the brain tissue, bisulfite sequencing of Copy 5 and Copyl brain exhibited the saturated status in their methylated region while WT brain exhibited fragmentary methylated CpG sites. The $18.06 \%$ methylation percentage of Copy 5 brain was more than the WT brain, $14.25 \%$ (Figure 4 ). In the heart tissues, bisulfite sequencing showed that the Copy2 heart had extremely hypermethylated percentage with $13.33 \%$ more than WT heart, 2.94\% (Figure 4). Particularly, the data showed the inhibition of Copy2 heart mRNA (Figure 1). In the liver, the different methylation pattern also appeared in the Copy4 liver relative to WT liver. Copy2 ear with 18.05\% methylation in the $O P N$ analyzed region was higher than the WT ear, 11.67\%; the result was proved by the experiment of OPN mRNA expression (Figure 1). These bisulfite sequencing results matched our previous hypothesis that methylation in 


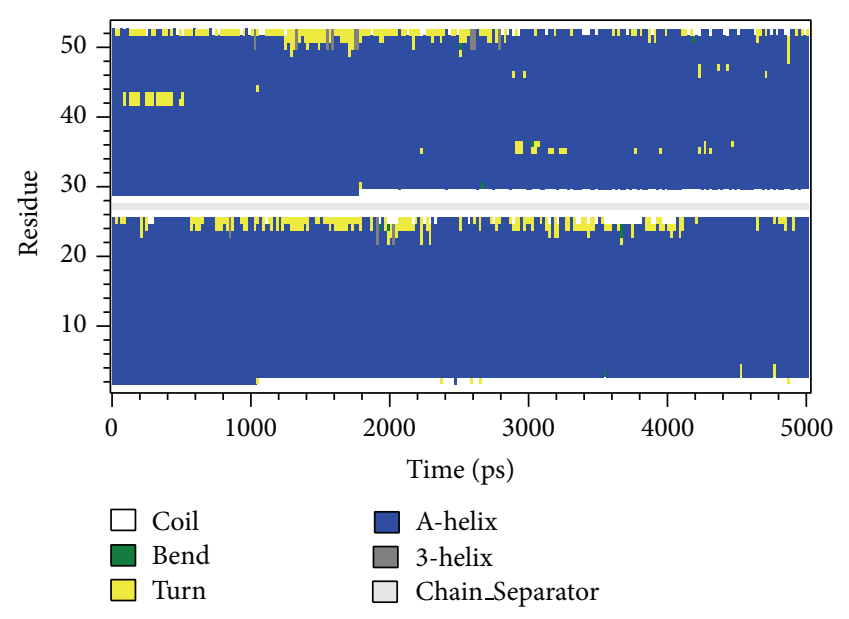

(a)

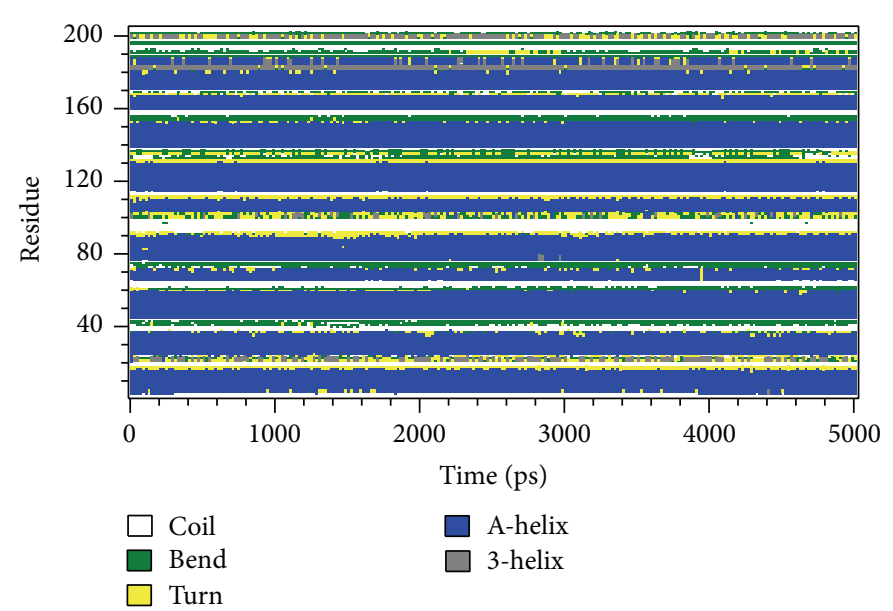

(b)

FIGURE 13: The secondary structure analysis for (a) c-Jun and (b) TFIIB over all simulation times. Each secondary type of the structure such as Bend, Turn, alpha helix (A-Helix), and $3_{10}$-helix (3-helix) is colored in green, yellow, blue, and gray, respectively. The "Chain_Seoarator" in (a) is used to differentiate between chain $\mathrm{A}$ and chain $\mathrm{B}$ of c-Jun.

the $O P N$ promoter region regulates the activity of $O P N$ promoter. Moreover, the hypermethylated OPN promoter may directly affect the activity of $O P N$ transcription, especially in heart and ear tissues.

\subsection{Analysis of Methylation Implication of OPN Transcription} by Promoter Assay. The analyzed OPN promoter region may involved with methylated control of gene transcription. Promoter assay was designed to explain the direct inhibition of $O P N$ promoter activity by methylation on the $O P N$ CpG sites. Four different truncated forms of $O P N$ full length $(2.6 \mathrm{~kb})$ were used to prove the hypothesis (Figure 5). Particularly, the truncated form $2.2 \mathrm{~kb}$ deleted the $377 \mathrm{bp}$ promoter region (-2615 -2239 $\mathrm{nt}$ ). This region is the analyzed region for bisulfite sequencing profile. And this region is thought to be the most possible element that regulates the OPN transcription. Moreover, four $\mathrm{CpG}$ sites were in vitro added to the methyl group by methyltransferase that can provide important evidence how methylation affect the $O P N$ transcription. Figure 5(b) shows that the OPN promoter activity was significantly decreased in methylated -2615-luc plasmid. However, -2239-luc that deleted the $377 \mathrm{bp}$ containing methylated characteristic DNA element leads to less inhibition of promoter activity than the methylated vector -2615 M-luc (Figures 5(a) and 5(b)). The results indicated that methylation in the front $O P N$ promoter is not only decreasing the promoter activity to the basal level but also recruiting the inhibition factors to enhance the inhibition ability. In order to avoid the effects of CpG sites in PGL3enhancer backbone, $-495 \mathrm{M}$-luc that have no methylated CpG sites in the OPN promoter but it can be methylated in the vector backbone CpG sites compared to -495-luc. Data showed that there is no difference in the promoter activity between methylation or unmethylation in the PGL3enhancer backbone CpG sites (Figure 5(b)). It is suggested that methylation in the critical region, such as OPN promoter front end, may lead to the rearrangement of chromatin structure. Otherwise, deletion of the OPN promoter to the $495 \mathrm{bp}$ with significant promotion of the promoter activity indicated that in the middle part of promoter DNA element may able to inhibit the activity of OPN promoter.

3.6. Methylation in CPG 13th and CpG 1st of OPN Promoter Blocks the Binding Access of Transcription Factors. We investigate that the methylated CpG sites in the -2615 -2239 nt of the OPN promoter region affect the transcription factor binding activity. Electrophoresis mobile shift assay was performed with nuclear extracts from human HEK293T and SH-SY5Y cell line. Four EMSA probes that contain the CpG 1, CpG 3-4, CpG 6-8, CpG 11-15, and CpG 19-20 were designed according to the $\mathrm{CpG}$ sites in our analyzed region (Figure 6). EMSA data suggested that CpG 13th and 1st sites showed methylation noncompetition phenomenon which had influence on binding with transcription factor (Figure 7). 19-20th CpG sites containing EMSA probe showed no competition ability in the methylated or unmethylated status. The premix with antibody and nuclear extracts by EMSA assay indicated that the c-Jun and c-Fos were involved in the binding to $\mathrm{CpG}$ sites 13 and 20 (Figure 8). However, the adding of antibody in the mixture of probe and nuclear extracts showed no significant shift bands. It is indicated that other transcription factors may also participate in the transcription activity OPN promoter. Thus, the c-Jun and c-Fos could be involved in the partial OPN transcriptional activity in a competition way. In addition, the c-Jun had higher binding affinity than cFos in the EMSA probe analysis. We therefore did followed computational survey to see why c-Juc affects the consequent $O P N$ transcription.

3.7. The Computational Biology Results. After surveying the possible zone for OPN promoter hypermethylation, we found that the AP1 (c-Jun) sequence frequently appeared in our 


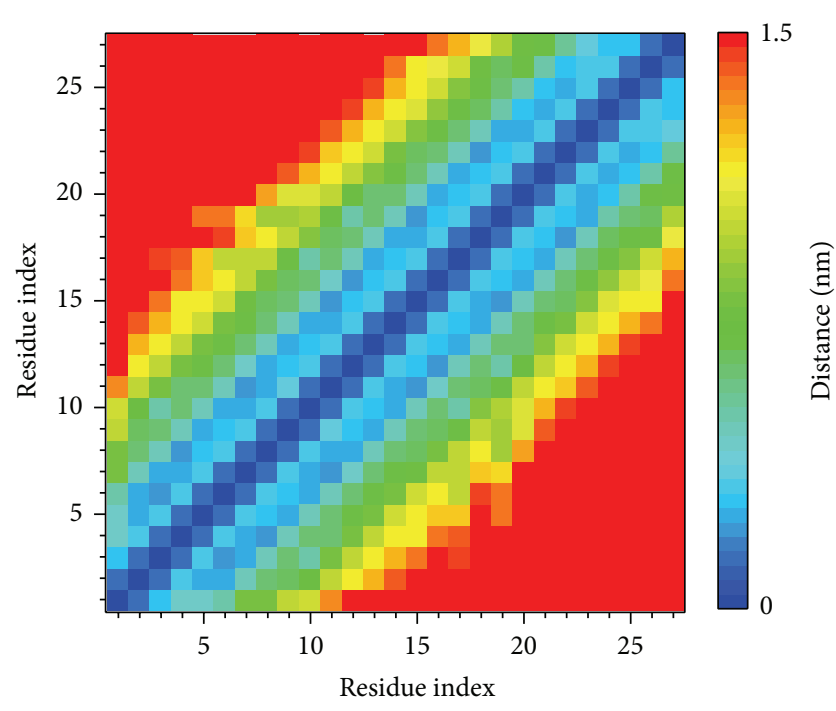

(a)

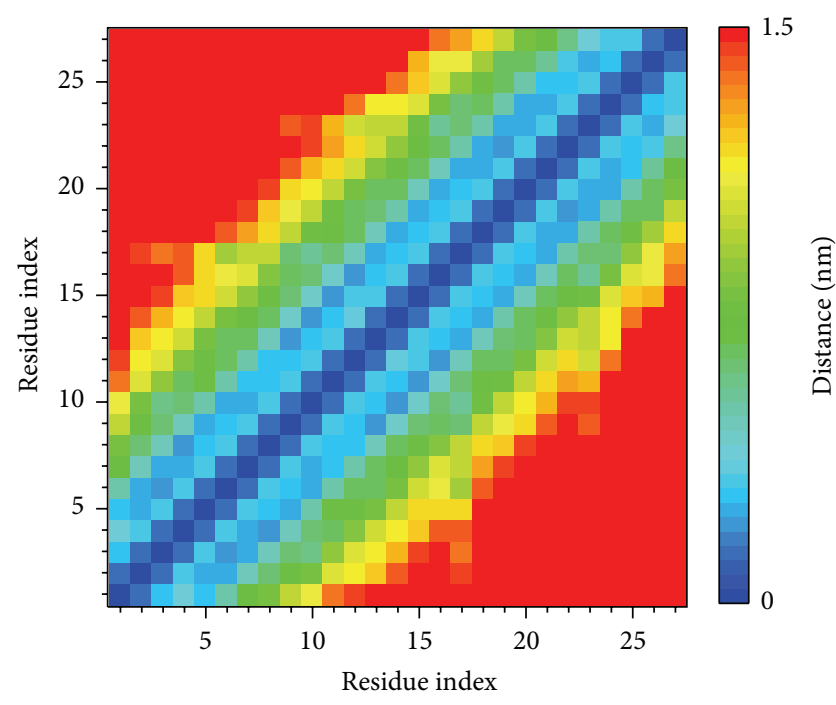

(b)

FIGURE 14: Matrices of smallest distance between each residue on (a) chain A of c-Jun and (b) chain B of c-Jun. The value of distance between residues is represented by rainbow bar, and the value of distance with longer than $1.5 \mathrm{~nm}$ is colored in red. The indexes of residues from 0 to 27 indicate residues from 254 to 280 on each chain of c-Jun.

molecular laboratory study. Then we further validated that the c-Jun methylation will cease the further mRNA production by inhibiting the binding of RNA-polymerase II initiation factor TFIIB. We do the further computational modelling for mechanism survey between the c-Jun and TFIIB. By the Z-DOCK analysis, we found the c-Jun and TFIIB could combine tightly (Figure 9). We chose the highest docking pose (dock score $=18.04$ ) for further MD analysis.

Then the disorder predication was employed to observe the protein folding analysis and the result is shown in Figure 10. We found that the c-Jun has relative high disorder in folding than TFIIB; we suppose that the flexibility of c-Jun structure could easier bound to TFIIB. This finding could be an explanation why the c-Jun bound to TFIIB by Z-DOCK program. To confirm the stability of the c-Jun and TFIIB complex, the series of molecular dynamic studies further visualize their interactions.

Protein complex RMSD analysis proved that the c-Jun and TFIIB were stable from 3000 ps to 5000 ps. In addition, we found the TFIIB are easier to be stable during the molecular dynamics (Figure 11(a)). We also found the radius of gyration tend to be stable for all simulation times with average of $2.05 \mathrm{~nm}$ (Figure 11(b)), suggesting that the two protein structures are compact after binding together. Figure 11(c) also shows the binding complex in a stable fluctuation and the energy of the binding complex is stable around $-9.15 \times$ $10^{5}(\mathrm{~kJ} / \mathrm{mol})$.

3.8. Stability Analysis of Residues on the Major Binding Region during MD Simulation. To analyze the flexibility of residues on protein structure, the RMSF calculation was used to observe the flexibility of each residue; Figure 12(a) shows that the chain A of c-Jun had high frequency of fluctuation (binding site 200-210 binding resides). However, the chain $\mathrm{B}$ of the $\mathrm{c}$-Jun has relative fewer frequency of fluctuation and the major binding region (from 228 to 240 residues) showed a less fluctuation as shown in Figure 12(b). Figure 12(c) reveals the binding regions (200-210 binding residues) for chain A of c-Jun that is more fluctuated and unstable compared to the binding regions (228-240) for chain B of c-Jun binding site in the TFIIB binding region. Figure 13 is the result for secondary structure variation calculated by DSSP analysis. Most of the main scaffold belong to alpha Helix; there are no significant changes during the whole MD simulation. All helices of the secondary structure for c-Jun and TFIIB binding remained stable during a 5,000 ps simulation time (Figure 13). We thereafter surveyed the distance between each residue of cJun for 5,000 ps. The variation of distances between residues in c-Jun chain is wider than the distances of residues in cJun chain B. Therefore, the chain B of c-Jun is more stable for TFIIB binding (Figure 14).

The hydrophobic area was then calculated by SASA in Figure 15(a); the value of hydrophobic area decreased during the last 1000 ps. This indicated the compactness of the c-Jun and TFIIB binding increased by the MD time period in our study. It is worthy to know that the distance between centrals of masses of c-Jun and TFIIB was decreasing more and more after time goes by in the 5000 ps survey (Figure 15(b)). In the migration analysis of c-Jun and TFIIB, the MSD was employed to count the migration of c-Jun and TFIIB. The c-Jun is more unstable than TFIIB during the binding interaction throughout the whole $\mathrm{MD}$ simulation in 5000 ps period (Figure 15(c)). Besides, we further utilized the principal component analysis (PCA) to measure all MD frames over all simulation times. The first two eigenvectors (PC1 and PC2) were shown in Figure 18; most of frames are ranged in the short range of eigenvalues -10 and 10 in PC1 (Figure 16(a)), and arranged in eigenvalues from -5 to 5 in PC2 (Figure 16(b)). The phase space comparing for PC1 and PC2 was shown in Figure 17; we found that each frame 


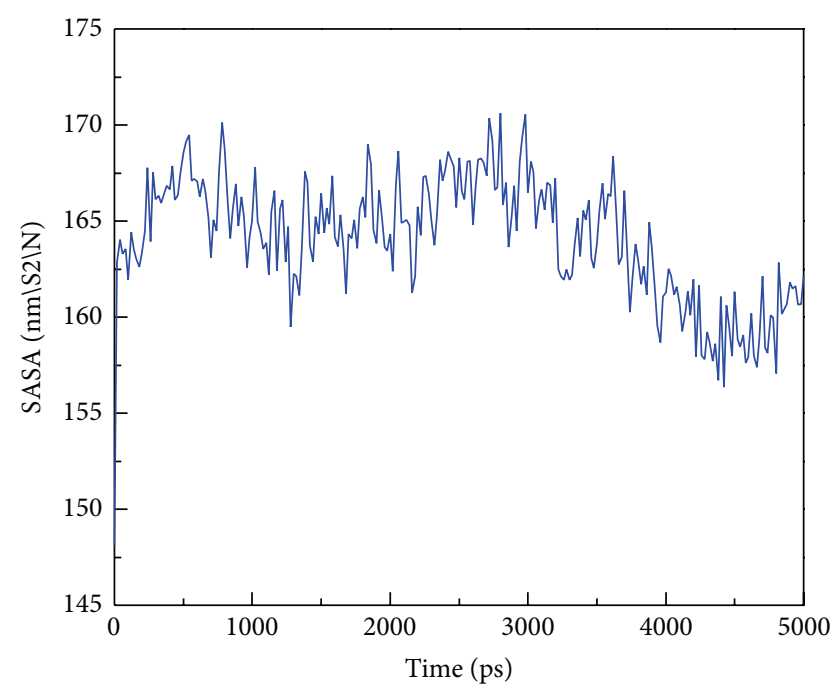

(a)

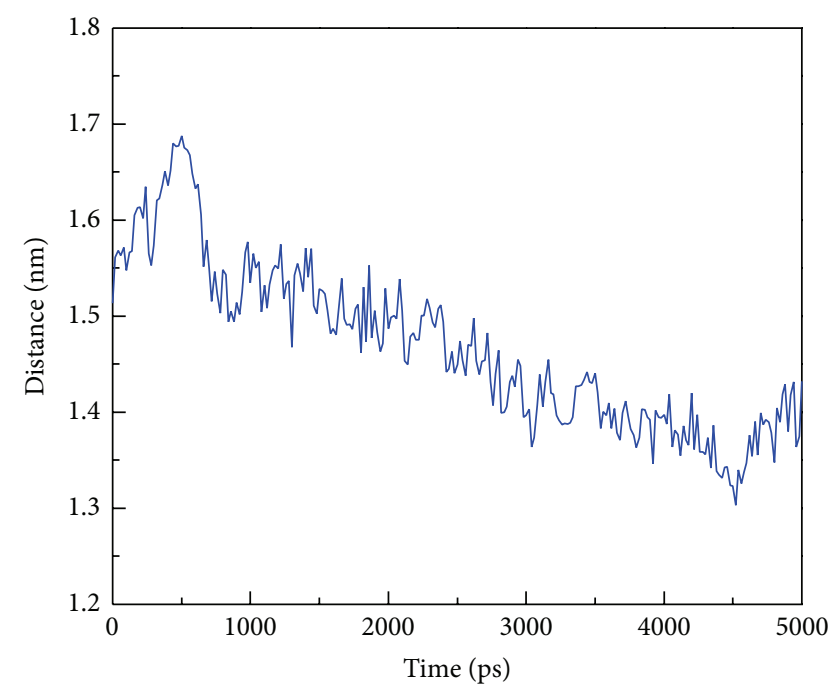

(b)

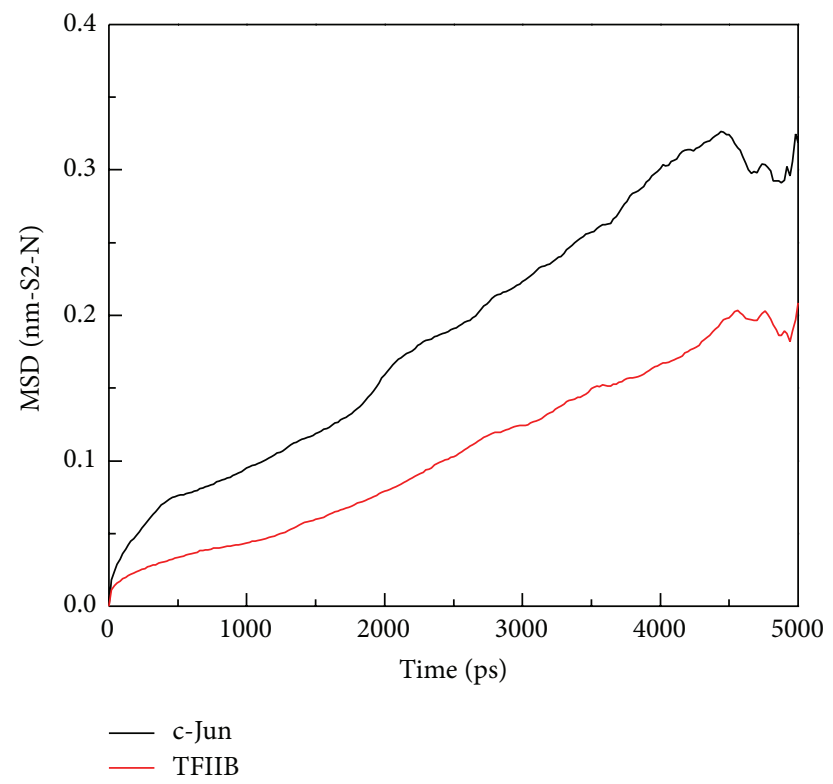

(c)

FIGURE 15: The area of solvent and protein migration analyses during simulation time of $5000 \mathrm{ps}$. (a) The total solvent accessible surface area of c-Jun and TFIIB complex; (b) the distance between the centrals of masses of of c-Jun and TFIIB; (c) trajectory analysis of MSD of c-Jun and TFIIB. The high values of MSD indicated the longer distance of migration from the initial binding position.

could be grouped into two clusters. This suggests that the motion of each frame was not changed significantly over all simulation times. In order to select the most representative structure for snapshot investigation, we did cluster analysis (Figure 18). We found that the last group (cluster 14) is the predominant cluster and is also displayed in the time range from 4000 to 5000 ps; the cluster 14 also appears most predominant in frame numbers (Figure 18), and the middle structure ( $4260 \mathrm{ps)}$ of cluster 14 is regarded as representative frame. For snapshot analysis, the comparison of initial and representative frames is shown in Figure 19; we found that the chain A of c-Jun is more encompassed by TFIIB at 4260 ps through the inward rotation of TFIIB. This made the bindings between chain A of c-Jun and TFIIB more compacted through 0 ps to 4260 ps along with time. The elevated activation between TFIIB and chain A of c-Jun is also confirmed in RMSF analysis. Therefore, we supposed the initiated transcription factor on RNA polymerase II (TFIIB) is closed interaction to the chain A of c-Jun (AP1) from 0 ps to 4260 ps. This hypothesis was also confirmed by Figure 20. There were more spaces between chain B and TFIIB than chain A. Hence, we could see that the TFIIB acts more close to 


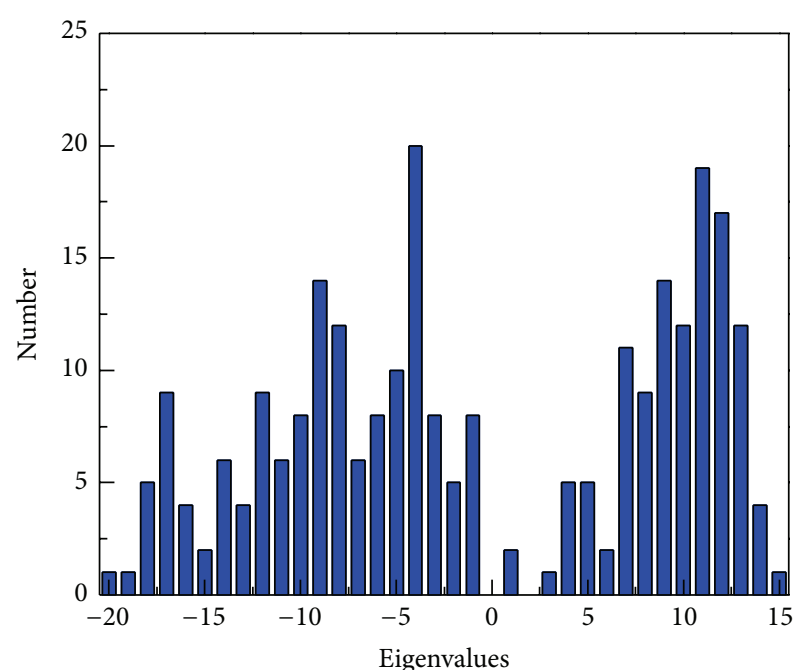

(a)

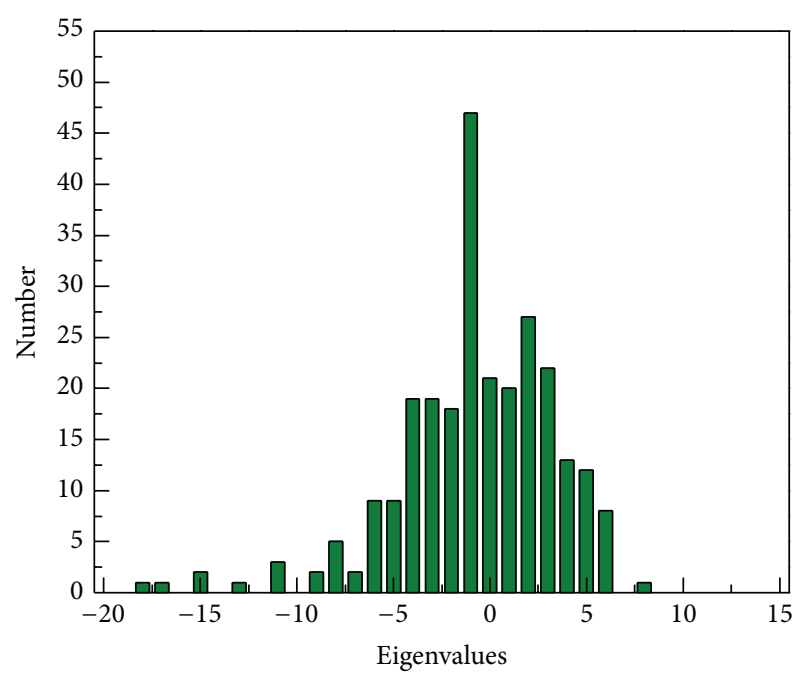

(b)

FIGURE 16: The number of MD frames of the first two eigenvectors (PC1 and PC2) by PCA analysis during simulation time of 5000 ps. The higher range of eigenvalue denotes the wider motion of protein structure over all simulation times.

the chain A of c-Jun (Figure 20). Overall, we presume that the initiation of $O P N$ transcription started from TFIIB binding to chain A of c-Jun.

\section{Discussion}

Previous study has shown that DNA element (GGGTCATATGGTTCA) located in osteopontin promoter -2245 to $-2259 \mathrm{nt}$ can be regulated by vitamin D3 [27]. This DNA regulation region can easily be affected by the change of calcium concentration. The promoter region of porcine $O P N$ was analyzed in transcription factor binding sites except the region -2615 to -2239 . Interestingly, this region of porcine $O P N$ promoter is rich in $\mathrm{CpG}$ sites compared to human, mouse, and bovine genome. Sakata also proved that

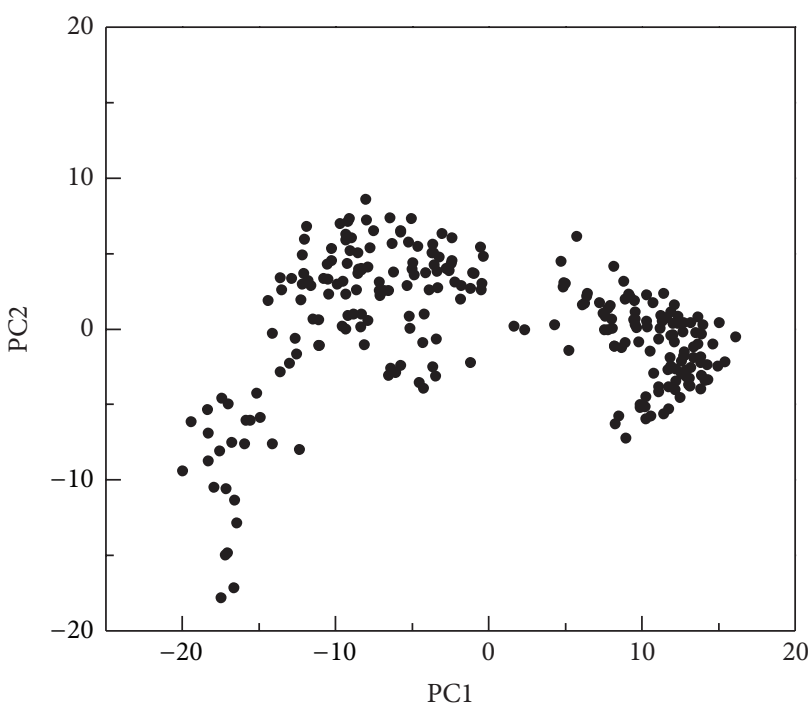

FIgURE 17: Phase space analysis of comparing the first two eigenvectors ( $\mathrm{PC1}$ and $\mathrm{PC} 2$ ) for principle component analysis. The eigenvalues of the two eigenvectors are projected into one phase space; small motion of protein structure could be grouped into clusters.

OPN promoter transcription activity is regulated by some specific DNA modification mechanism of rearrangement of chromatin structure [22].

In the present study, four cloned pigs were surrounded by many defects. For example, Copyl pig had a retardation of limb bone growth. Copy2 heart organ showed a pericarditis and copy 3 heart had valvular heart disease. This physiology defects appeared aberrant development, especially in bone or heart, may involved in the initially fetus stage with inappropriate organ differentiate. Thus, our data suggested that the consequent result in aberrant $O P N$ expression or incompletely epigenetic modification in $O P N$ promoter (Figure 5). These aberrant molecular data of OPN are correlated with the defects of bone and heart in cloned pigs. Semiquantitative PCR of OPN mRNA showed that discrepant expression pattern was identified in several cloned pig tissues, especially in brain (99.75\% up-regulation), heart (11.5\% downregulation), and ear (18.03\% downregulation) (Figure 2). OPN mRNA has different expression in brain development in different embryonic stages [28]. The overexpression of OPN in brain tissue may cause some unexpected brain damage or neuron development. $O P N$ can induce myocardial fibrosis and repair tissue after inflammation. Lacking $O P N$ will cause faulty wound healing after myocardial infarction $[29,30]$. Silent expression of OPN in cloned pig' heart tissue may also be the main cause of heart disease.

Recent studies indicated that OPN gene expression may be affected by treatment of TSA (a histone deacetylase inhibitor). The results applied that $O P N$ promoter could be regulated by epigenetic mechanism [22]. In this study, we investigated $O P N$ methylation profile after 5 -aza-dc treatment. The results indicated that mRNA expression of $O P N$ is 

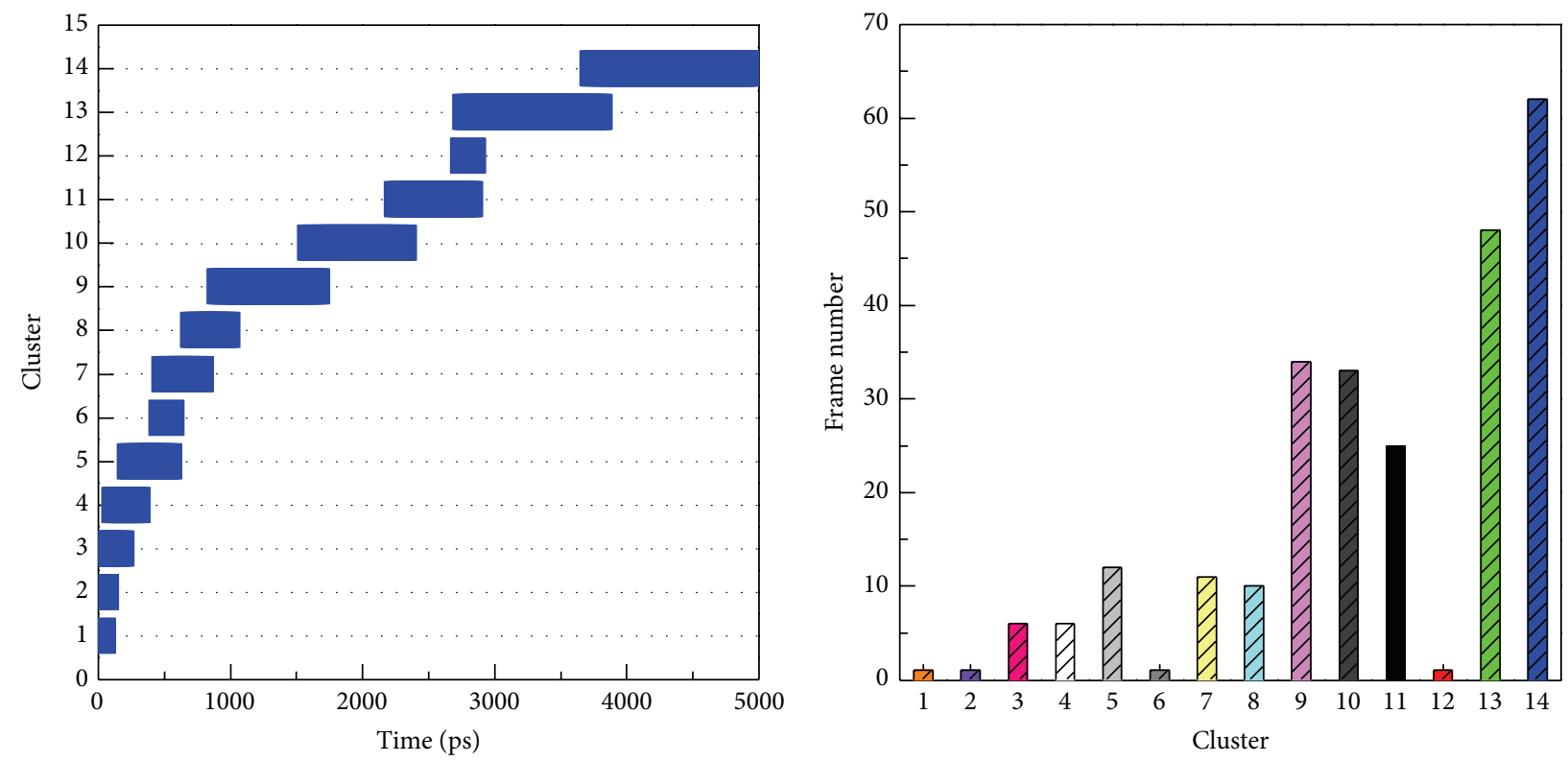

Figure 18: Cluster analysis of all MD frames of c-Jun and TFIIB complex during simulation time of 5 ns for identifying representative structure. All MD frames were grouped into fourteen clusters by linkage method; the RMSD cut-off distance between each neighbor frame is $0.14 \mathrm{~nm}$. The most predominant group is the cluster 14, which is displaced in the region of simulation time from 4000 to 5000 ps, and the MD frames in cluster 14 are the most number among all clusters. The middle frame of cluster 14 is displaced in simulation time of 4260 ps.

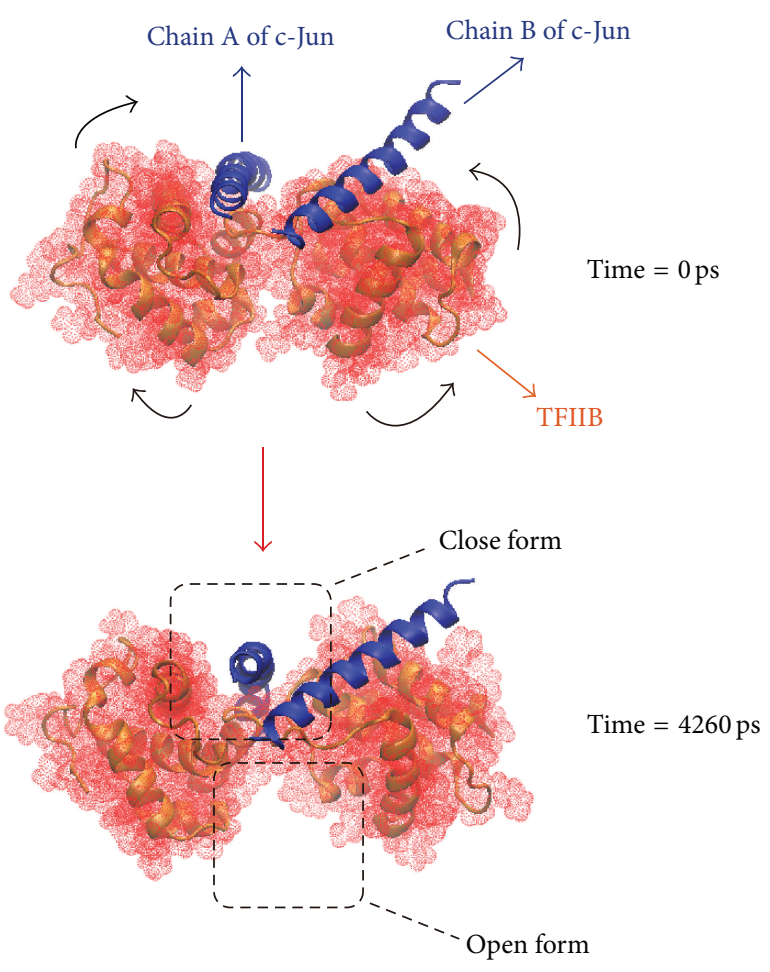

FIGURE 19: The structural comparison between the first frame $(0 \mathrm{ps})$ and representative structure $(4260 \mathrm{ps})$. The structures of cJun and TFIIB are represented by blue ribbon and red solid phase, respectively. The chain A of c-Jun was surrounded more compactly by TFIIB at $4260 \mathrm{ps}$ by the structural inward rotation to make more compactness between TFIIB and c-Jun through 0 ps to 4260 ps.

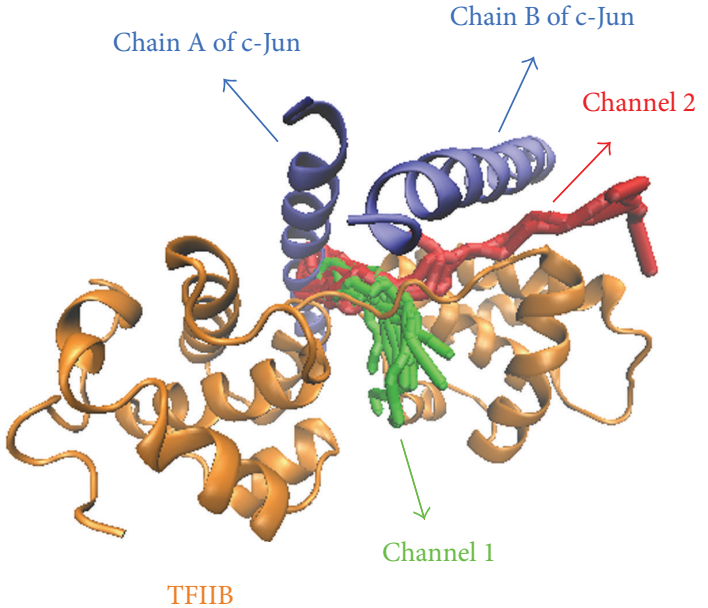

FIgURE 20: The possible space prediction between c-Jun and TFIIB among all simulation times. The predicted channels are colored in red and green. The structures of c-Jun and TFIIB are colored in blue and orange, respectively. Each possible space is represented by channels; each channel was generated by Caver 3.0 program.

directly affected by adding methyltransferase inhibitor 5-azadc (Figure 4). COBRA was performed to study the methylation of OPN front end promoter in wild-type and cloned pig different tissues. Sodium bisulfite sequencing analysis also revealed that the methylation of $\mathrm{CpG}$ sites concentrated in front of the $20 \mathrm{CpG}$ sites in front of OPN promoter (Figure 5). Discrepancy methylation in this promoter region 
also happened in brain, heart, ear, and liver tissues between wild-type and cloned pigs (Figure 5). It revealed that DNA methylation of $O P N$ promoter may be involved with regulation of expression of $O P N$ mRNA. In order to characterise which promoter DNA element is important, four constructs of OPN promoter (-2615-luc, -2239-luc, -1505-luc, and -495-luc) were used for analysis. Obvious downregulation in methylated -2615-luc (HpaII and HhaI methyltransferase) was observed. Compared to the deletion of this control region $(\sim 390 \mathrm{nt})$ in front of OPN promoter, the decreasing level of promoter activity is not as obvious as $-2615 \mathrm{M}$ luc construct (Figure 7). It means that methylation in the front of OPN promoter caused some silent mechanism that make chromosome structure more compact or block some promotion transcription factors. The EMSA data indicated that 13th $\mathrm{CpG}$ site of our analyzed region could bind to AP1 transcription factor and binding activity is affected by methylation in this CpG site (Figure 7). Taken together, all these findings correlated with DNA methylation in tissueor cell-specific gene expression. OPN promoter region was densely methylated in some low expression (Figures 2, 4, and 5).

Our data revealed that DNA methylation of $\mathrm{CpG}$ sites in $O P N$ promoter was the main mechanism through specific transcription factor that makes the tissue-specific expression. In previous study, AP1-like binding site (TGAGCGA) was identified as a methylated insulator region in human blastoma cell line [31]. Analyzed region in front of porcine OPN promoter showed that $\mathrm{CpG}$ 1st binding site contained the specific binding site sequence. While in our interesting DNA regulation region range from -2615 to $-2239 \mathrm{nt}$ of OPN promoter also exhibited little block access in the competition of probe. It is suggested that $\mathrm{CpG} 1$ st and $\mathrm{CpG}$ 13th play an important role in methylation controlled mechanism to regulate gene expression. We finally utilize Z-dock program [32] to analyze the interaction between AP1 (PDB code: 1JNM) and RNA polymerase II initial transcription factor (TFIIB) (PDB code: 1VOL) [33] to see if they had stable binding. From the docking result of Z-dock (Figure 8), we proved the AP1 is significantly bound to TFIIB. We also found the AP1 can autoregulate the HDAC-1 in promoter region and lead to significant higher degrees of hypermethylation in the $O P N$ promoter region and cause AP1 to be hypermethylated consequently ceasing the OPN mRNA expression [34].

Further mechanical studies by the computational biology also pointed out that the DNA sequence for hypermethylation of OPN promoter binding sites is c-Jun. The chain A of cJun could be encompassed more tightly by inward rotational structure change of TFIIB during the MD process (Figure 19). Therefore, we found c-Jun had crucial role for interaction of initiating transcription by RNA polymerase II. The methylation of c-Jun leads to of hyper-condense helix structural change and makes transcription termination which stops $O P N$ mRNA production. Therefore, the MD docking results reconfirm the c-Jun partake the crucial roles in consequent $O P N$ transcription that matches our wet laboratory studies. We suppose this will cause the problems in the embryonic development and lead to threatened conditions. Therefore, adjusting OPN promoter c-Jun (AP1) methylation will affect transcription binding and could be the treatment for genetic developing errors in the future.

In conclusion, aberrant methylation of porcine $O P N$ gene was frequently found in different tissues of somatic nuclear transferred cloning pigs and bisulfite sequence data suggested that the OPN promoter region of -2615 to $-2239 \mathrm{nt}$ may be a crucial regulation DNA element. In pig ear fibroblast cell culture study, the demethylation of OPN promoter was found in dose-dependent response of 5-aza-dc treatment and followed the OPN mRNA reexpression. In cloned pig study, discrepant expression pattern was identified in several cloned pig tissues, especially in brain (99.75\% up-regulation), heart (11.5\% down-regulation), and ear (18.03\% downregulation). Promoter assay data revealed that four methylated CpG sites presenting in the -2615 to -2239 nt region cause significant downregulation (approximately 75\%) of OPN promoter activity $(P<0.001)$. EMSA data also suggested that $\mathrm{CpG}$ 13 th and 1st sites showed methylation noncompetition phenomenon which had influence on binding with transcription factor.

\section{Conflict of Interests}

The authors declare that there is no conflict of interests regarding the publication of this paper.

\section{Authors' Contribution}

Chih-Jie Shen, Yung-An Tsou, and Hsiao-Ling Chen contributed equally to this work.

\section{Acknowledgments}

This research was supported by Grants NSC-95-2313-B005-030-MY3, NSC-101-2313-B-005-014-MY3, 101-2314-B039-013-MY3, NSC102-2325-B039-001, and NSC102-2221-E468-027- from the National Science Council and was partly supported by the Ministry of Education, Taiwan, under the aiming top university plan (ATU-101-s0508). The authors would like to thank their colleagues (Drs. Tung-Chou Tsai, Cheng-Wei Lai, and Zi-Lun Lai) in the Molecular Embryology \& DNA Methylation Laboratory for their help with discussions and technical issues. This study is also supported in part by Taiwan Department of Health Clinical Trial and Research Center of Excellence (DOH102-TD-B-111-004), Taiwan Department of Health Cancer Research Center of Excellence (MOHW103-TD-B-111-03), and CMU under the Aim for Top University Plan of the Ministry of Education, Taiwan.

\section{References}

[1] Y. M. Leung, Y. M. Wong, S. W. Chen et al., "Down-regulation of voltage-gated $\mathrm{Ca}^{2+}$ channels in $\mathrm{Ca}^{2+}$ store-depleted rat insulinoma RINm5F cells," BioMedicine, vol. 3, no. 3, pp. 130139, 2013. 
[2] M.-C. Lin, S.-Y. Tsai, F.-Y. Wang et al., "Leptin induces cell invasion and the upregulation of matrilysin in human colon cancer cells," BioMedicine, vol. 3, no. 4, pp. 174-180, 2013.

[3] Y.-M. Chang, B. K. Velmurugan, W. W. Kuo, and et al, "Inhibitory effect of alpinate Oxyphyllae fructus extracts on Ang IIinduced cardiac pathological remodeling-related pathways in H9c2 cardiomyoblast cells," BioMedicine, vol. 3, no. 4, pp. 148-152, 2013.

[4] T. H. Bestor, "The DNA methyltransferases of mammals," Human Molecular Genetics, vol. 9, no. 16, pp. 2395-2402, 2000.

[5] P. H. Tate and A. P. Bird, "Effects of DNA methylation on DNA-binding proteins and gene expression," Current Opinion in Genetics and Development, vol. 3, no. 2, pp. 226-231, 1993.

[6] W. H. Eyestone and K. H. Campbell, "Nuclear transfer from somatic cells: applications in farm animal species." Journal of Reproduction and Fertility, vol. 54, pp. 489-497, 1999.

[7] T. Wakayama, A. C. F. Perry, M. Zuccotti, K. R. Johnson, and R. Yanagimachi, "Full-term development of mice from enucleated oocytes injected with cumulus cell nuclei," Nature, vol. 394, no. 6691, pp. 369-374, 1998.

[8] T. Wakayama and R. Yanagimachi, "Mouse cloning with nucleus donor cells of different age and type," Molecular Reproduction and Development, vol. 58, no. 4, pp. 376-383, 2001.

[9] J. Ohgane, T. Wakayama, Y. Kogo et al., "DNA methylation variation in cloned mice," Genesis, vol. 30, no. 2, pp. 45-50, 2001.

[10] G. A. Johnson, R. C. Burghardt, F. W. Bazer, and T. E. Spencer, "Osteopontin: roles in implantation and placentation," Biology of Reproduction, vol. 69, no. 5, pp. 1458-1471, 2003.

[11] G. F. Weber, S. Zawaideh, S. Hikita, V. A. Kumar, H. Cantor, and S. Ashkar, "Phosphorylation-dependent interaction of osteopontin with its receptors regulates macrophage migration and activation," Journal of Leukocyte Biology, vol. 72, no. 4, pp. 752761, 2002.

[12] M. Mazzali, T. Kipari, V. Ophascharoensuk, J. A. Wesson, R. Johnson, and J. Hughes, "Osteopontin-a molecule for all seasons," QJM, vol. 95, no. 1, pp. 3-13, 2002.

[13] N. S. Fedarko, A. Jain, A. Karadag, M. R. van Eman, and L. W. Fisher, "Elevated serum bone sialoprotein and osteopontin in colon, breast, prostate, and lung cancer," Clinical Cancer Research, vol. 7, no. 12, pp. 4060-4066, 2001.

[14] G. Chakraborty, S. Jain, and G. C. Kundu, "Osteopontin promotes vascular endothelial growth factor-dependent breast tumor growth and angiogenesis via autocrine and paracrine mechanisms," Cancer Research, vol. 68, no. 1, pp. 152-161, 2008.

[15] R. Agnihotri, H. C. Crawford, H. Haro, L. M. Matrisian, M. C. Havrda, and L. Liaw, "Osteopontin, a novel substrate for matrix metalloproteinase-3 (stromelysin-1) and matrix metalloproteinase-7 (matrilysin)," The Journal of Biological Chemistry, vol. 276, no. 30, pp. 28261-28267, 2001.

[16] D. Wang, S. Yamamoto, N. Hijiya, E. N. Benveniste, and C. L. Gladson, "Transcriptional regulation of the human osteopontin promoter: Functional analysis and DNA-protein interactions," Oncogene, vol. 19, no. 50, pp. 5801-5809, 2000.

[17] H. Rangaswami, A. Bulbule, and G. C. Kundu, "Osteopontin: role in cell signaling and cancer progression," Trends in Cell Biology, vol. 16, no. 2, pp. 79-87, 2006.

[18] H. Rangaswami, A. Bulbule, and G. C. Kundu, "Nuclear factorinducing kinase plays a crucial role in osteopontin-induced $\mathrm{MAPK} / \mathrm{I} \kappa \mathrm{B} \alpha$ kinase-dependent nuclear factor $\kappa \mathrm{B}$-mediated promatrix metalloproteinase- 9 activation," Journal of Biological Chemistry, vol. 279, no. 37, pp. 38921-38935, 2004.
[19] A. B. Tuck, D. M. Arsenault, F. P. O’Malley et al., "Osteopontin induces increased invasiveness and plasminogen activator expression of human mammary epithelial cells," Oncogene, vol. 18, no. 29, pp. 4237-4246, 1999.

[20] H. Okamoto, “Osteopontin and cardiovascular system," Molecular and Cellular Biochemistry, vol. 300, no. 1-2, pp. 1-7, 2007.

[21] F. Schoensiegel, R. Bekeredjian, A. Schrewe et al., "Atrial natriuretic peptide and osteopontin are useful markers of cardiac disorders in mice," Comparative Medicine, vol. 57, no. 6, pp. 546553, 2007.

[22] R. Sakata, S. Minami, Y. Sowa, M. Yoshida, and T. Tamaki, "Trichostatin A activates the osteopontin gene promoter through AP1 site," Biochemical and Biophysical Research Communications, vol. 315, no. 4, pp. 959-963, 2004.

[23] K. H. Choi, H. Basma, J. Singh, and P. W. Cheng, "Activation of CMV promoter-controlled glycosyltransferase and $\beta$ galactosidase glycogenes by butyrate, tricostatin $\mathrm{A}$, and 5 -aza$2^{\prime}$-deoxycytidine," Glycoconjugate Journal, vol. 22, no. 1-2, pp. 63-69, 2005.

[24] B. M. Kumar, H.-F. Jin, J.-G. Kim et al., "DNA methylation levels in porcine fetal fibroblasts induced by an inhibitor of methylation, 5-azacytidine," Cell and Tissue Research, vol. 325, no. 3, pp. 445-454, 2006.

[25] S. Pronk, S. Páll, R. Schulz et al., "GROMACS 4.5: a highthroughput and highly parallel open source molecular simulation toolkit," Bioinformatics, vol. 29, no. 7, pp. 845-854, 2013.

[26] E. Chovancova, A. Pavelka, P. Benes et al., "CAVER 3.0: a tool for the analysis of transport pathways in dynamic protein structures," PLoS Computational Biology, vol. 8, no. 10, Article ID e1002708, 2012.

[27] Q. Zhang, J. L. Wrana, and J. Sodek, "Characterization of the promoter region of the porcine opn (osteopontin, secreted phosphoprotein 1) gene. Identification of positive and negative regulatory elements and a "silent" second promoter," European Journal of Biochemistry, vol. 207, no. 2, pp. 649-659, 1992.

[28] M. Y. Lee, J. S. Choi, S. W. Lim, J. H. Cha, M. H. Chun, and J. W. Chung, "Expression of osteopontin mRNA in developing rat brainstem and cerebellum," Cell and Tissue Research, vol. 306, no. 2, pp. 179-185, 2001.

[29] L. Liaw, D. E. Birk, C. B. Ballas, J. S. Whitsitt, J. M. Davidson, and B. L. Hogan, "Altered wound healing in mice lacking a functional osteopontin gene (spp1)," The Journal of Clinical Investigation, vol. 101, no. 7, pp. 1468-1478, 1998.

[30] N. A. Trueblood, Z. Xie, C. Communal et al., "Exaggerated left ventricular dilation and reduced collagen deposition after myocardial infarction in mice lacking osteopontin," Circulation Research, vol. 88, no. 10, pp. 1080-1087, 2001.

[31] M. Fujimoto, R. Kitazawa, S. Maeda, and S. Kitazawa, "Methylation adjacent to negatively regulating AP-1 site reactivates TrkA gene expression during cancer progression," Oncogene, vol. 24, no. 32, pp. 5108-5118, 2005.

[32] Accelerys, Discovery Studio Client v2.5, Accelrys, San Diego, Calif, USA, 2009.

[33] D. B. Nikolov, H. Chen, E. D. Halay et al., "Crystal structure of a TFIIB-TBP-TATA-element ternary complex," Nature, vol. 377, no. 6545, pp. 119-128, 1995.

[34] P. Sharma, S. Kumar, and G. C. Kundu, “Transcriptional regulation of human osteopontin promoter by histone deacetylase inhibitor, trichostatin A in cervical cancer cells," Molecular Cancer, vol. 9, article 178, 2010. 

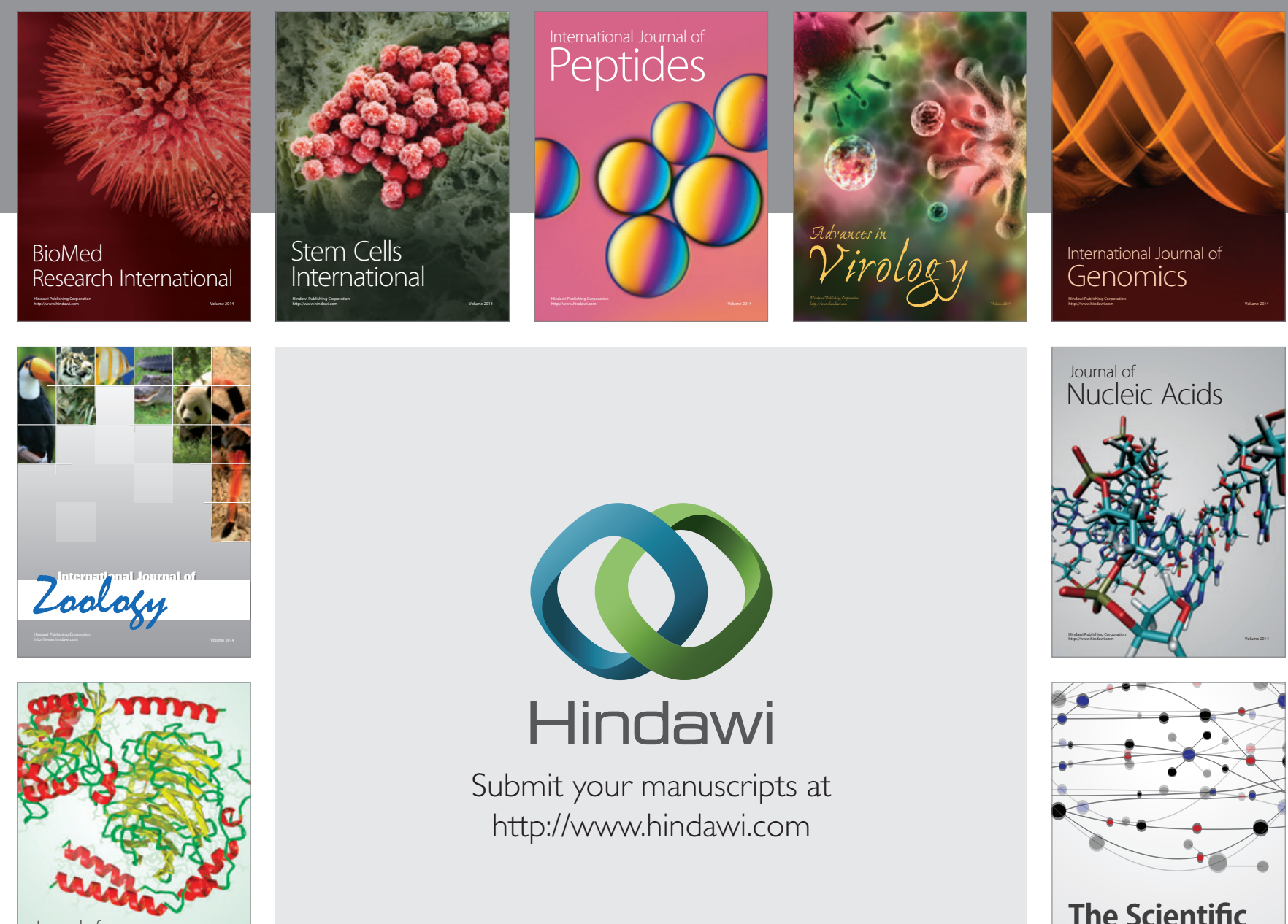

Submit your manuscripts at

http://www.hindawi.com

Journal of
Signal Transduction
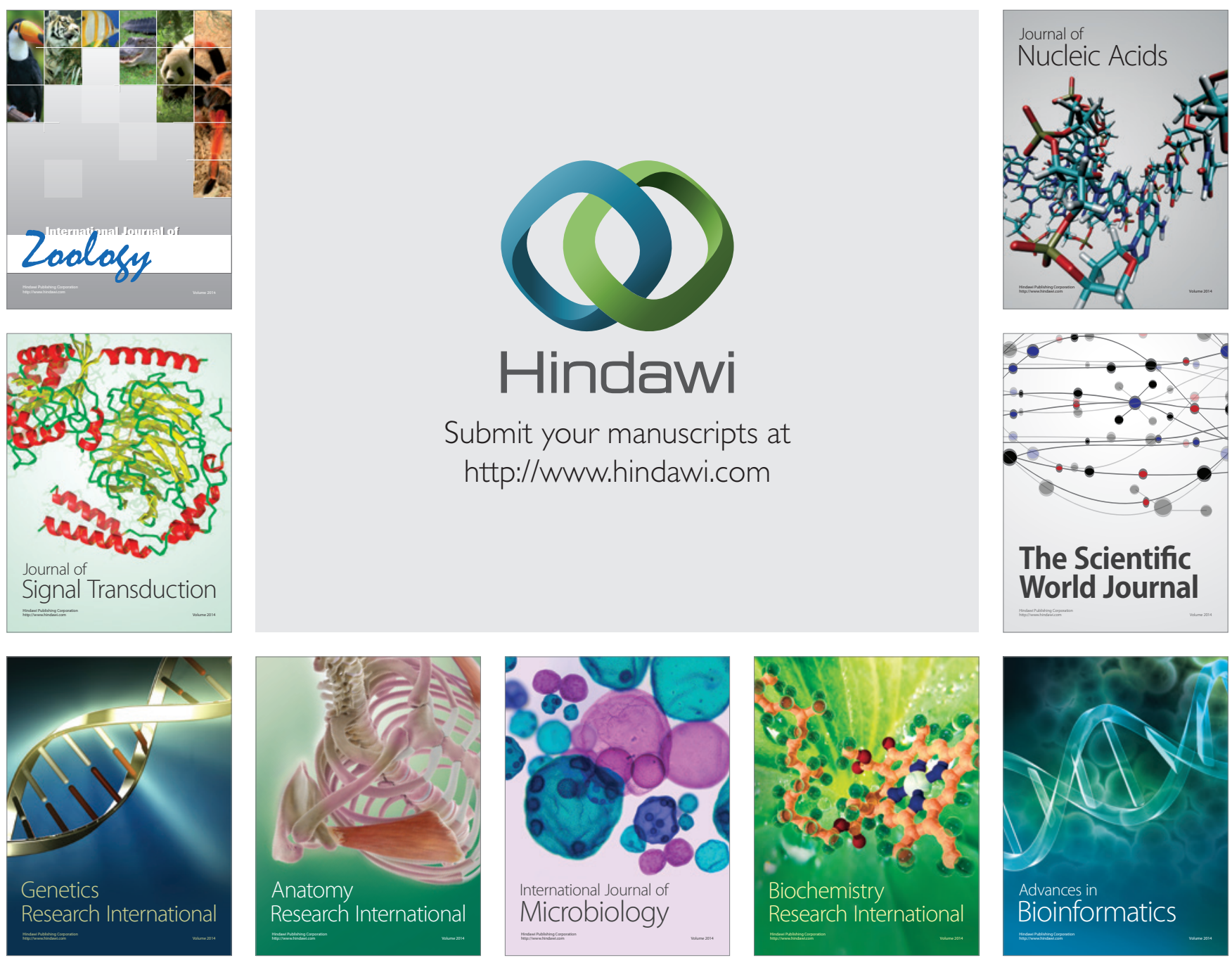

The Scientific World Journal
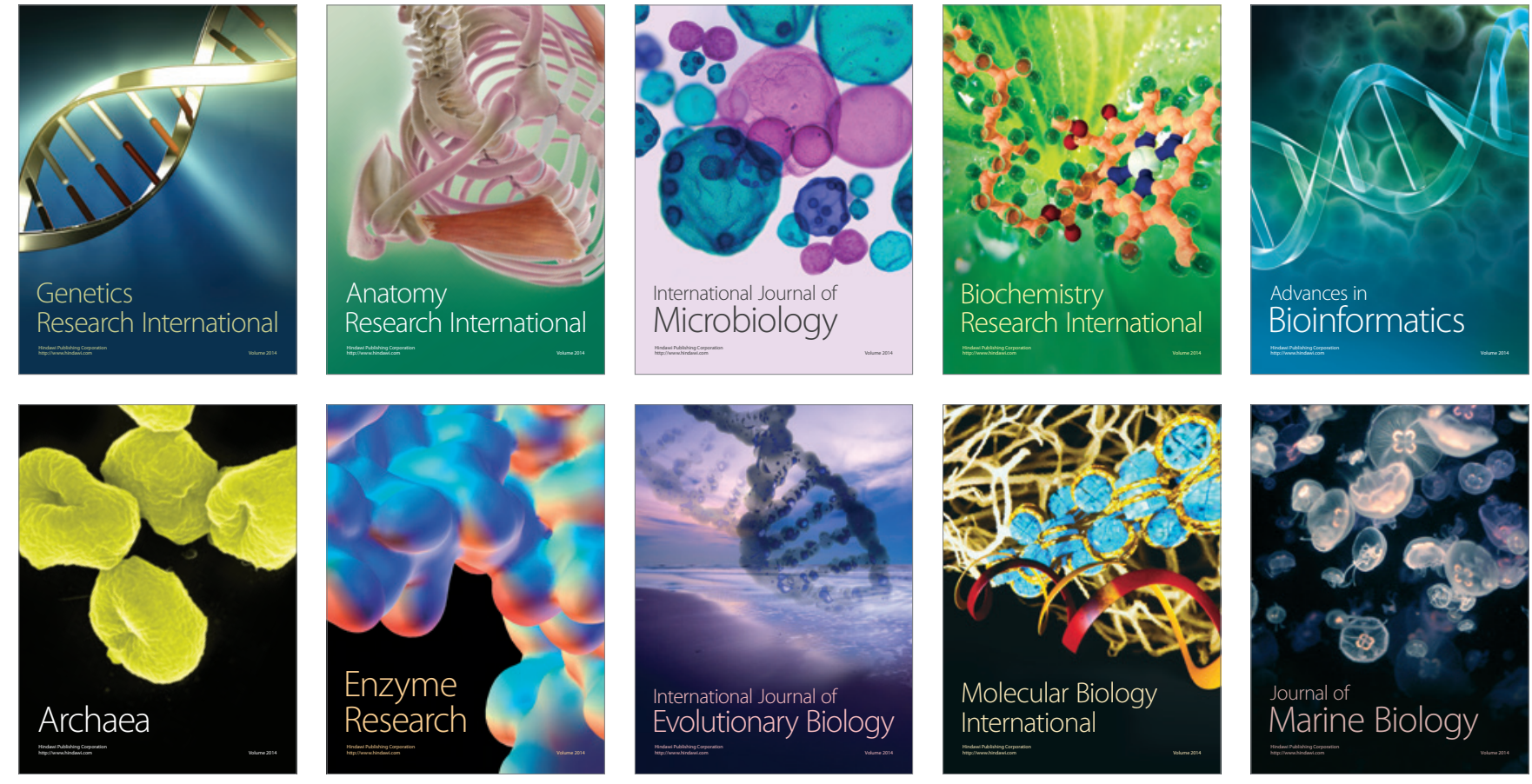